\title{
A FLEXIBLE NONPARAMETRIC TEST FOR CONDITIONAL INDEPENDENCE
}

\author{
Meng HuAng \\ Freddie Mac \\ YIXIAO SUN AND HALBERT WHITE \\ UC San Diego
}

\begin{abstract}
This paper proposes a nonparametric test for conditional independence that is easy to implement, yet powerful in the sense that it is consistent and achieves $n^{-1 / 2}$ local power. The test statistic is based on an estimator of the topological "distance" between restricted and unrestricted probability measures corresponding to conditional independence or its absence. The distance is evaluated using a family of Generically Comprehensively Revealing (GCR) functions, such as the exponential or logistic functions, which are indexed by nuisance parameters. The use of GCR functions makes the test able to detect any deviation from the null. We use a kernel smoothing method when estimating the distance. An integrated conditional moment (ICM) test statistic based on these estimates is obtained by integrating out the nuisance parameters. We simulate the critical values using a conditional simulation approach. Monte Carlo experiments show that the test performs well in finite samples. As an application, we test an implication of the key assumption of unconfoundedness in the context of estimating the returns to schooling.
\end{abstract}

\section{INTRODUCTION}

In this paper, we propose a flexible nonparametric test for conditional independence. Let $X, Y$, and $Z$ be three random vectors. The null hypothesis we want to test is that $Y$ is independent of $X$ given $Z$, denoted by

$Y \perp X \mid Z$.

Intuitively, this means that given the information in $Z, X$ cannot provide additional information useful in predicting $Y$. Dawid (1979) showed that some simple heuristic properties of conditional independence can form a conceptual framework for many important topics in statistical inference: sufficiency and ancillarity, parameter identification, causal inference, prediction sufficiency, data

We thank Graham Elliott, Dimitris Politis, Patrick Fitzsimmons, Jin Seo Cho, Liangjun Su, James Hamilton, Andres Santos, Brendan Beare, and seminar participants at the University of California San Diego, Hong Kong University of Science and Technology, Peking University Guanghua school of management, and Bates White, LLC for helpful comments and suggestions. We are equally grateful for the constructive comments from Yuichi Kitamura, the co-editor, and two anonymous referees. Special thanks to Liangjun Su for sharing computer programs. Address correspondence to Meng Huang, Freddie Mac, 1551 Park Run Dr., McLean, VA 22102, USA; email: nkhuangmeng@gmail.com or to Yixiao Sun, Department of Economics 0508, University of California, San Diego, La Jolla, CA 92093, USA; email: yisun@ucsd.edu. 
selection mechanisms, invariant statistical models, and a subjectivist approach to model-building.

An important application of conditional independence testing in economics is to test a key assumption identifying causal effects. Suppose we are interested in estimating the effect of $X$ (e.g., schooling) on $Y$ (e.g., income), and that $X$ and $Y$ are related by the equation

$Y=\theta_{0}+\theta_{1} X+U$

where $U$ (e.g., ability) is an unobserved cause of $Y$ (income) and $\theta_{0}$ and $\theta_{1}$ are unknown coefficients, with $\theta_{1}$ representing the effect of $X$ on $Y$. (We write a linear structural equation here merely for concreteness.) Since $X$ is typically not randomly assigned and is correlated with $U$ (e.g., unobserved ability will affect both schooling and income), OLS will generally fail to consistently estimate $\theta_{1}$. Nevertheless, if, as in Griliches and Mason (1972) and Griliches (1977), we can find a set of covariates $Z$ (e.g., proxies for ability, such as AFQT scores) such that

\section{$U \perp X \mid Z$,}

we can estimate $\theta_{1}$ consistently by various methods: covariate adjustment, matching, methods using the propensity score such as weighting and blocking, or combinations of these approaches.

The assumption in (1) is a key assumption for identifying $\theta_{1}$. It is called a conditional exogeneity assumption by White and Chalak (2008). It enforces the "ignorability" or "unconfoundedness" condition, also known as "selection on observables" (Barnow, Cain, and Goldberger, 1981).

Note that the conditional independence assumption in (1) cannot be directly tested since $U$ is unobservable. But if there are other observable covariates $V$ satisfying certain conditions (see White and Chalak, 2010), we have

\section{$U \perp X \mid Z \quad$ implies $\quad V \perp X \mid Z$,}

so we can test the assumption in (1) by testing its implication, $V \perp X \mid Z$. Section 6 of this paper applies this test in the context of a nonparametric study of returns to schooling.

In the literature, there are many tests for conditional independence when the variables are categorical. However, in economic applications it is common to condition on continuous variables, and there are only a few nonparametric tests for the continuous case. Previous work on testing conditional independence for continuous random variables includes Linton and Gozalo (1997, "LG"), Fernandes and Flores (1999, "FF"), and Delgado and Gonzalez-Manteiga (2001, "DG"). Su and White have several papers (2003, 2007, 2008, 2010, "SW") addressing this question. Although SW's tests are consistent against any deviation from the null, they are only able to detect local alternatives converging to the null at a rate slower than $n^{-1 / 2}$ and hence suffer from the "curse of dimensionality." We will compare our test with the LG, DG, and SW tests in our simulation study. 
Recently, Song (2009) has proposed a distribution-free conditional independence test of two continuous random variables given a parametric single index that achieves the local $n^{-1 / 2}$ rate. Specifically, Song (2009) tests the hypothesis

$Y \perp X \mid \lambda_{\theta}(Z)$

where $\lambda_{\theta}(\cdot)$ is a scalar-valued function known up to a finite-dimensional parameter $\theta$, which must be estimated.

A main contribution here is that our proposed test also achieves $n^{-1 / 2}$ local power, despite its fully nonparametric nature. In contrast to Song (2009), the conditioning variables can be multi-dimensional. The test is motivated by a series of papers on consistent specification testing by Bierens (1982, 1990), Bierens and Ploberger (1997), and Stinchcombe and White (1998, "StW"), among others. Whereas Bierens (1982, 1990) and Bierens and Ploberger (1997) construct the integrated conditional moment (ICM) tests that essentially compare a restricted parametric model with an unrestricted regression model, the test in this paper follows a suggestion of $\mathrm{StW}$, which is based on the estimates of the topological distance between unrestricted and restricted probability measures, corresponding to conditional independence or its absence.

This distance is measured indirectly by a family of moments, which are the differences of the expectations under the null and under the alternative for a set of test functions. The chosen test functions make use of Generically Comprehensively Revealing (GCR) functions, such as the logistic or normal cumulative distribution functions (CDFs), and are indexed by a continuous nuisance parameter vector $\gamma$. Under the null, all moments are zeroes. Under the alternative, the moments are nonzero for essentially all choices of $\gamma$. This is in contrast with DG (2001), which employs an indicator testing function that is not generically and comprehensively revealing.

We estimate these moments by their sample analogs, using kernel smoothing. An ICM test statistic based on these is obtained by integrating out the nuisance parameters. Its limiting null distribution is a functional of a mean zero Gaussian process. We simulate critical values using a conditional simulation approach suggested by Hansen (1996) in a different setting.

Our GCR approach requires bounded random variables. When any random variable is not bounded, we first standardize it on the basis of estimated location and scale parameters and then apply a bounded and invertible transformation to the standardized data. The location and scale parameters can be the mean and standard deviation or other more robust measures such as the median and interquartile range, respectively.

The plan of the paper is as follows. In Section 2, we specify a family of moment conditions which is (essentially) equivalent to the null hypothesis of conditional independence and forms a basis for our test. In Section 3, we establish stochastic approximations of the empirical moment conditions uniformly over the nuisance parameters. We derive the finite-dimensional weak convergence of the empirical moment process. We also provide a bandwidth choice for practical use: a simple 
"plug-in" estimator of the MSE-optimal bandwidth. In Section 4, we formally introduce and analyze our ICM test statistic. In Section 5, we report some Monte Carlo results, examining the size and power properties of our test and comparing its performance with that of a variety of other tests in the literature. In Section 6, we study the returns to schooling, using the proposed statistic to test an implication of the key assumption of unconfoundedness. The last section concludes. The appendix contains the proofs of the main results and shows that the estimation errors in the location and scale parameters have no impact on our asymptotic theory.

\section{THE NULL HYPOTHESIS AND THE TESTING APPROACH}

\subsection{The Null Hypothesis}

Let $X, Y$, and $Z$ be three random vectors, with dimensions $d_{X}, d_{Y}$, and $d_{Z}$, respectively. Denote $W=\left(X^{\prime}, Y^{\prime}, Z^{\prime}\right) \in \mathbb{R}^{d}$ with $d=d_{X}+d_{Y}+d_{Z}$. Given an IID sample $\left\{X_{i}, Y_{i}, Z_{i}\right\}_{i=1}^{n}$, we want to test the null that $Y$ is independent of $X$ conditional on $Z$, i.e.,

$H_{0}: Y \perp X \mid Z$

against the alternative that $Y$ and $X$ are dependent conditional on $Z$, i.e.,

$H_{a}: Y \not \perp X \mid Z$.

Let $F_{Y \mid X Z}(y \mid x, z)$ be the conditional distribution function of $Y$ given $(X, Z)=$ $(x, z)$ and $F_{Y \mid Z}(y \mid z)$ be the conditional distribution function of $Y$ given $Z=z$. Then we can express the null as

$F_{Y \mid X Z}(y \mid x, z)=F_{Y \mid Z}(y \mid z)$.

The following three expressions are equivalent to one another and to (3):

$F_{X \mid Y Z}(x \mid y, z)=F_{X \mid Z}(x \mid z)$

$F_{X Y \mid Z}(x, y \mid z)=F_{X \mid Z}(x \mid z) F_{Y \mid Z}(y \mid z)$,

$F_{X Y Z}(x, y, z) F_{Z}(z)=F_{X Z}(x, z) F_{Y Z}(y, z)$,

where we have used the standard notations for distribution functions.

The approach adopted in this paper is inspired by a series of papers on consistent specification testing: Bierens (1982, 1990), Bierens and Ploberger (1997), and StW (1998), among others. The tests in those papers are based on an infinite number of moment conditions indexed by nuisance parameters. Consider, as an example, the conditional mean function $g(x)=E(Y \mid X=x)$. Bierens (1990) tests the hypothesis that the parametric functional form, $f(x, \lambda)$, is correctly 
specified in the sense that $g(x)=f\left(x, \theta_{0}\right)$ for some $\theta_{0} \in \Theta$. The test statistic is based on an estimator of a family of moments $E\left[\left(Y-f\left(X, \theta_{0}\right)\right) e^{\gamma^{\prime} X}\right]$ indexed by a nuisance parameter vector $\gamma$. Under the null hypothesis of correct specification, these moments are zeroes for all $\gamma$. Bierens's (1990) Lemma 1 shows that the converse essentially holds, due to the properties of the exponential function, making the test capable of detecting all deviations from the null. The similar idea is used in Bierens and Wang (2012) for testing a parametric specification of the conditional distribution function.

$\mathrm{StW}$ find that a broader class of functions has this property. They extend Bierens's result by replacing the exponential function in the moment conditions with any GCR function, and by extending the probability measures considered in the Bierens (1990) approach to signed measures. As stated in StW, GCR functions include nonpolynomial real analytic functions, e.g., exp, logistic CDF, sine, cosine, and also some nonanalytic functions like the normal CDF or its density. Further, they point out that such specification tests are based on estimates of topological distances between a restricted model and an unrestricted model. Following this idea, we can construct a test for conditional independence based on estimates of a topological distance between unrestricted and restricted probability measures corresponding to conditional independence or its absence.

To define the GCR property formally, let $\mathcal{C}(F)$ be the set of continuous functions on a compact set $F \subset \mathbb{R}^{d}$, and $s p\left[H_{\varphi}\right]$ be the linear span of a collection of functions $H_{\varphi}(\Gamma)$. We write $\tilde{w}:=\left(1, w^{\prime}\right)^{\prime}$. The definition below is the same as Definition 3.6 in StW.

DEFINITION 1 (StW, Definition 3.6). We say that $H_{\varphi}=\left\{H: \mathbb{R}^{d} \rightarrow\right.$ $\left.\mathbb{R} \mid H(w)=\varphi\left(\tilde{w}^{\prime} \gamma\right), \gamma \in \Gamma \subset \mathbb{R}^{1+d}\right\}$ is generically comprehensively revealing if for all $\Gamma$ with nonempty interior, the uniform closure of $s p\left[H_{\varphi}\right]$ contains $\mathcal{C}(F)$ for every compact set $F \subset \mathbb{R}^{d}$.

Intuitively, GCR functions are a class of functions indexed by $\gamma \in \Gamma$ whose span comes arbitrarily close to any continuous function, regardless of the choice of $\Gamma$, as long as it has nonempty interior. When there is no confusion, we simply call $\varphi$ GCR if the generated $H_{\varphi}$ is GCR.

We now establish an equivalent hypothesis in the form of a family of moment conditions following StW. Let $P$ be the joint distribution of the random vector $W$, and let $Q$ be the joint distribution of $W$ with $Y \perp X \mid Z$. Thus, $P$ is an unrestricted probability measure, whereas $Q$ is restricted. To be specific, $P$ and $Q$ are defined such that for any event $A$,

$$
P(A) \equiv \int_{A} d F_{X Y Z}(x, y, z)=\int_{A} d F_{X Y \mid Z}(x, y \mid z) d F_{Z}(z)
$$

and

$$
Q(A) \equiv \int_{A} d F_{X \mid Z}(x \mid z) d F_{Y \mid Z}(y \mid z) d F_{Z}(z) .
$$


Note that the measure $P$ will be the same as the measure $Q$ if and only if the null is true:

$P(A)=\int_{A} d F_{X Y \mid Z}(x, y \mid z) d F_{Z}(z) \stackrel{H_{0}}{=} \int_{A} d F_{X \mid Z}(x \mid z) d F_{Y \mid Z}(y \mid z) d F_{Z}(z)=Q(A)$

for all Borel sets $A$. Testing the null hypothesis is thus equivalent to testing whether there is any deviation of $P$ from $Q$. It should be pointed out that the marginal distribution of $Z$ is the same under $P$ and $Q$ regardless of whether the null is true or not.

Let $E_{P}$ and $E_{Q}$ be the expectation operators with respect to the measure $P$ and the measure $Q$. Define

$$
\Delta_{\varphi}(\gamma) \equiv E_{P}\left[\varphi\left(\tilde{W}^{\prime} \gamma\right)\right]-E_{Q}\left[\varphi\left(\tilde{W}^{\prime} \gamma\right)\right]
$$

where $\gamma \equiv\left(\gamma_{0}, \gamma_{1}^{\prime}, \gamma_{2}^{\prime}, \gamma_{3}^{\prime}\right)^{\prime} \in \mathbb{R}^{1+d}$ is a vector of nuisance parameters, $\tilde{W}=$ $\left(1, W^{\prime}\right)^{\prime}$, and $\varphi$ is such that the indicated expectations exist for all $\gamma$. Under the null hypothesis, $\Delta_{\varphi}(\gamma)$ is obviously zero for any choice of $\gamma$ and any choice of $\varphi$, including GCR functions. To construct a powerful test, we want $\Delta_{\varphi}(\gamma)$ to be nonzero under the alternative. If $\Delta_{\varphi_{0}}\left(\gamma_{0}\right)$ is not zero under some alternative, we say that $\varphi_{0}$ can detect that particular alternative for the choice $\gamma=\gamma_{0}$. An arbitrary function $\varphi_{0}$ may fail to detect some alternatives for some choices of $\gamma$. Nevertheless, according to $\mathrm{StW}$, if $W$ is a bounded random vector, the properties of GCR functions imply that they can detect all possible alternatives for essentially all $\gamma \in \Gamma \subset \mathbb{R}^{1+d}$ with $\Gamma$ having nonempty interior. "Essentially all" $\gamma \in \Gamma$ means that the set of "bad" $\gamma$ 's, i.e., the set $\left\{\gamma \in \Gamma: \Delta_{\varphi}(\gamma)=0\right.$ and $\left.Y \not \perp X \mid Z\right\}$ has Lebesgue measure zero and is not dense in $\Gamma$.

Given that any deviation of $P$ from $Q$ can be detected by essentially any choice of $\gamma \in \Gamma$, testing $H_{0}: Y \perp X \mid Z$ is equivalent to testing

$$
H_{0}: \Delta_{\varphi}(\gamma)=0 \text { for essentially all } \gamma \in \Gamma
$$

for a GCR function $\varphi$ and a set $\Gamma$ with nonempty interior. The alternative is $H_{a}$ : $H_{0}$ is false.

A straightforward testing approach is to estimate $\Delta_{\varphi}(\gamma)$ and to see how far the estimate is from zero. However, there are two technical issues. First, the result of StW requires $W$ to be bounded. To achieve the boundedness, we can replace each element $V$ of $W$ by $\Psi_{V}(V)$, where $\Psi_{V}$ is a bounded one-to-one mapping with Borel measurable inverse. Define $\Psi_{Y}(Y)=\left(\Psi_{Y_{1}}\left(Y_{1}\right), \ldots, \Psi_{Y_{d_{Y}}}\left(Y_{d_{Y}}\right)\right)^{\prime}$ and define $\Psi_{X}(X)$ and $\Psi_{Z}(Z)$ similarly. Then $Y \perp X \mid Z$ is equivalent to $\Psi_{Y}(Y) \perp$ $\Psi_{X}(X) \mid \Psi_{Z}(Z)$. The equivalence holds because the sigma fields are not affected by the transformation. So it is innocuous to assume that $W$ has a bounded support.

In practice, we recommend choosing a bounded set, say $[a, b]^{d}$, to closely match the support of the GCR function we use. Depending on whether the random variables have bounded supports or not, we can achieve this using a different 
transformation $\Psi$. As an example, suppose that the support of $V$ is a bounded interval, say $\left[v_{\min }, v_{\max }\right]$ for some known finite constants $v_{\min }$ and $v_{\max }$. Then we can take

$$
\Psi_{V}(V)=a+(b-a) \frac{\left(V-v_{\min }\right)}{v_{\max }-v_{\min }},
$$

which obviously meets our requirement. If the end points of the support are not known, we can estimate them by $\hat{v}_{\min }=\min _{i=1, \ldots, n}\left(V_{i}\right)$ and $\hat{v}_{\max }=$ $\max _{i=1, \ldots, n}\left(V_{i}\right)$ and plug the estimates into (10). Under some mild conditions, $\hat{v}_{\min }$ and $\hat{v}_{\text {max }}$ converge to the true endpoints at the fast rate of $1 / n$. As a result, we can show that the estimation uncertainty has no effect on our asymptotic results.

When the random variable $V$ has an unbounded support, we first standardize it and then take

$$
\Psi_{V}(V)=a+(b-a) \frac{\arctan \left(\left(V-\mu_{v}\right) / \sigma_{v}\right)+0.5 \pi}{\pi},
$$

where $\mu_{v}$ and $\sigma_{v}$ are location and scale parameters. For example, $\mu_{v}$ and $\sigma_{v}$ can be the mean and standard deviation of $V$. By construction, $\Psi_{V}(V) \in[a, b]$. We can also use other bounded functions such as $\Psi_{V}(V)=a+(b-a) F\left(\left(V-\mu_{v}\right) / \sigma_{v}\right)$ for a CDF $F$. The standardization ensures that $\Psi_{V}(V)$ does not reside in a small subset of $[a, b]$. See Bierens and Wang (2012) for more discussion. When $\mu_{v}$ and $\sigma_{v}$ are not known, we can estimate them by $\hat{\mu}_{v}$ and $\hat{\sigma}_{v}$ respectively and plug them into (11). In Section A.2, we make the transformation explicit and show that under some mild conditions including the $\sqrt{n}$-consistency of $\hat{\mu}_{v}$ and $\hat{\sigma}_{v}$, the estimator errors in $\hat{\mu}_{v}$ and $\hat{\sigma}_{v}$ have no impact on the asymptotic properties of our proposed test. Here for notational simplicity we leave this transformation implicit and assume that $P\left(W \in[a, b]^{d}\right)=1$. Without loss of generality, we let $a=0$ and $b=1$ for our theoretical development.

The second issue is related to the nonparametric estimation of $\Delta_{\varphi}(\gamma)$. It involves a nonparametric estimator $\hat{f}_{Z}$ of the density $f_{Z}$ in the denominator of the test statistic, making the analysis of limiting distributions awkward. To avoid this technical issue, we compute the expectations of $\varphi f_{Z}$ rather than those of $\varphi$, leading to a new "distance" metric between $P$ and $Q$ :

$$
\Delta_{\varphi f}(\gamma)=E_{P}\left[\varphi\left(\tilde{W}^{\prime} \gamma\right) f_{Z}(Z)\right]-E_{Q}\left[\varphi\left(\tilde{W}^{\prime} \gamma\right) f_{Z}(Z)\right]
$$

Using the change-of-measure technique, we have

$$
\Delta_{\varphi f}(\gamma)=C\left\{E_{P^{*}}\left[\varphi\left(\tilde{W}^{\prime} \gamma\right)\right]-E_{Q^{*}}\left[\varphi\left(\tilde{W}^{\prime} \gamma\right)\right]\right\},
$$

where $P^{*}$ and $Q^{*}$ are probability measures defined according to

$$
\begin{aligned}
P^{*}(A) & =\int_{A} f_{Z}(z) d F_{X Y \mid Z}(x, y \mid z) d F_{Z}(z) / C \text { and } \\
Q^{*}(A) & =\int_{A} f_{Z}(z) d F_{X \mid Z}(x \mid z) d F_{Y \mid Z}(y \mid z) d F_{Z}(z) / C
\end{aligned}
$$


with $C=\int f_{Z}^{2}(z) d z$ being the normalizing constant. Under the null of $H_{0}: Y \perp$ $X \mid Z, P^{*}$ and $Q^{*}$ are the same measure, and so $\Delta_{\varphi f}(\gamma)=0$ for all $\gamma \in \Gamma$. Under the alternative of $H_{a}: Y \not \perp X \mid Z, P^{*}$ and $Q^{*}$ are different measures. By definition, if $\varphi$ is GCR, then its revealing property holds for any probability measure (see Definition 3.2 of StW). So under the alternative, we have $\Delta_{\varphi f}(\gamma) \neq 0$ for essentially all $\gamma \in \Gamma$. The behaviors of $\Delta_{\varphi f}(\gamma)$ under $H_{0}$ and $H_{a}$ imply that we can employ $\Delta_{\varphi f}(\gamma)$ in place of $\Delta_{\varphi}(\gamma)$ to perform our test.

To sum up, when $\varphi$ is a GCR function, $W$ has bounded supports, $\Gamma$ has nonempty interior, and $\int f_{Z}^{2}(z) d z<\infty$, a null hypothesis equivalent to conditional independence is

$H_{0}: \Delta_{\varphi f}(\gamma)=0$ for essentially all $\gamma \in \Gamma$.

That is, the null hypothesis of conditional independence is equivalent to a family of moment conditions indexed by $\gamma$. For notational simplicity, we drop the subscript and write $\Delta(\gamma):=\Delta_{\varphi f}(\gamma)$ hereafter.

\subsection{Heuristics for Rates}

When the probability density functions exist, the conditional independence is equivalent to any of the following:

$$
\begin{aligned}
f_{Y \mid X Z}(y \mid x, z) & =f_{Y \mid Z}(y \mid z), \\
f_{X \mid Y Z}(x \mid y, z) & =f_{X \mid Z}(x \mid z), \\
f_{X Y \mid Z}(x, y \mid z) & =f_{X \mid Z}(x \mid z) f_{Y \mid Z}(y \mid z), \\
f_{X Y Z}(x, y, z) f_{Z}(z) & =f_{X Z}(x, z) f_{Y Z}(y, z),
\end{aligned}
$$

where the notation for density functions is self-explanatory. One way to test conditional independence is to compare the densities in a given equation to see if the equality holds. For example, Su and White's (2008) test essentially compares $f_{X Y Z} f_{Z}$ with $f_{X Z} f_{Y Z}$. To do that, they estimate $f_{X Y Z}, f_{Z}, f_{X Z}$, and $f_{Y Z}$ nonparametrically, so their test has power against local alternatives at a rate of only $n^{-1 / 2} h^{-d / 4}$, the slowest rate of the four nonparametric density estimators, i.e., the rate for $\hat{f}_{X Y Z}$. This rate is slower than $n^{-1 / 2}$ and hence reflects the "curse of dimensionality." The dimension here is $d=d_{X}+d_{Y}+d_{Z}$, which is at least three and could potentially be larger.

To achieve the rate $n^{-1 / 2}$, we do not compare the density functions directly. Instead, our family of moment conditions indirectly measures the distance between $f_{X Y Z} f_{Z}$ and $f_{X Z} f_{Y Z}$, so that for each given $\gamma$, the test statistic is based on an estimator of an average that can achieve an $n^{-1 / 2}$ rate, just as a semiparametric estimator would.

To better understand the moment conditions of the equivalent null, we write

$$
\begin{aligned}
\Delta(\gamma)= & \int \varphi\left(\tilde{w}^{\prime} \gamma\right) f_{Z}(z) f_{X Y Z}(x, y, z) d x d y d z \\
& -\int \varphi\left(\tilde{w}^{\prime} \gamma\right) f_{Y Z}(y, z) f_{X Z}(x, z) d x d y d z .
\end{aligned}
$$


Instead of comparing $f_{X Y Z} f_{Z}$ with $f_{Y Z} f_{X Z}$, we now compare their integral transforms. Before the transformation, $f_{X Y Z} f_{Z}$ and $f_{Y Z} f_{X Z}$ are functions of $(x, y, z)$, the data points, and those functions can only be estimated at a nonparametric rate slower than $n^{-1 / 2}$. But their integral transforms are now functions of $\gamma$. For each $\gamma$, the transformation is an average of the data so that semiparametric techniques could be used here to get an $n^{-1 / 2}$ rate. Essentially, we compare two functions by comparing their weighted averages. The two comparisons are equivalent because of the properties of the chosen test functions. That is, if we choose GCR functions for our test functions, defined on a compact index space $\Gamma$ with nonempty interior, and we do not detect any difference between $P^{*}$ and $Q^{*}$ transforms at essentially any point $\gamma$, then $P^{*}$ and $Q^{*}$ must agree, and as a consequence $P$ and $Q$ must agree. We gain robustness by integrating over many points $\gamma$.

\subsection{Empirical Moment Conditions}

With some abuse of notation, we write $\varphi\left(\gamma_{0}+x^{\prime} \gamma_{1}+y^{\prime} \gamma_{2}+z^{\prime} \gamma_{3}\right) \equiv \varphi(x, y, z ; \gamma)$. Define

$g_{X Z}(x, z ; \gamma)=E[\varphi(x, Y, z ; \gamma) \mid Z=z]=\int \varphi(x, y, z ; \gamma) f_{Y \mid Z}(y \mid z) d y$.

Then the moment conditions can be rewritten as

$\Delta(\gamma)=E\left[\varphi(X, Y, Z ; \gamma) f_{Z}(Z)\right]-E\left[g_{X Z}(X, Z ; \gamma) f_{Z}(Z)\right]$

The first term of $\Delta(\gamma)$ is a mean of $\varphi f_{Z}$, where $\varphi$ is known and $f_{Z}$ can be estimated by a kernel smoothing method. The second term is a mean of $g_{X Z} f_{Z}(Z)$, where the function $g_{X Z}(x, z ; \gamma)$ is a conditional expectation that can be estimated by a Nadaraya-Watson estimator:

$$
\hat{g}_{X Z}(x, z ; \gamma)=\frac{\sum_{j=1}^{n} \varphi\left(x, Y_{j}, z ; \gamma\right) K_{h}\left(Z_{j}-z\right)}{\sum_{j=1}^{n} K_{h}\left(Z_{j}-z\right)} .
$$

Thus we can estimate $\Delta(\gamma)$ by

$$
\begin{aligned}
\hat{\Delta}_{n, h}(\gamma)= & \frac{1}{n-1} \sum_{i=1}^{n}\left[\varphi\left(\tilde{W}_{i}^{\prime} \gamma\right) \hat{f}_{Z}\left(Z_{i}\right)\right]-\frac{1}{n-1} \sum_{i=1}^{n} \hat{g}_{X Z}\left(X_{i}, Z_{i} ; \gamma\right) \hat{f}_{Z}\left(Z_{i}\right) \\
= & \frac{1}{n-1} \sum_{i=1}^{n}\left[\varphi\left(\tilde{W}_{i}^{\prime} \gamma\right) \frac{1}{n} \sum_{j=1}^{n} K_{h}\left(Z_{j}-Z_{i}\right)\right. \\
& -\frac{1}{n(n-1)} \sum_{i=1}^{n} \sum_{j=1}^{n} \varphi\left(X_{i}, Y_{j}, Z_{i} ; \gamma\right) K_{h}\left(Z_{j}-Z_{i}\right)
\end{aligned}
$$




$$
\begin{aligned}
& =\frac{1}{n(n-1)} \sum_{i=1}^{n} \sum_{j=1}^{n}\left[\varphi\left(\tilde{W}_{i}^{\prime} \gamma\right)-\varphi\left(\tilde{W}_{i, j}^{\prime} \gamma\right)\right] K_{h}\left(Z_{j}-Z_{i}\right) \\
& =\frac{1}{n(n-1)} \sum_{i=1}^{n} \sum_{j=1, j \neq i}^{n}\left\{\left[\varphi\left(\tilde{W}_{i}^{\prime} \gamma\right)-\varphi\left(\tilde{W}_{i, j}^{\prime} \gamma\right)\right] K_{h}\left(Z_{i}-Z_{j}\right)\right\}
\end{aligned}
$$

where $\tilde{W}_{i, j}^{\prime} \gamma=\gamma_{0}+X_{i}^{\prime} \gamma_{1}+Y_{j}^{\prime} \gamma_{2}+Z_{i}^{\prime} \gamma_{3}$ and $K_{h}(u)$ is a multivariate kernel function. In this paper, we follow the standard practice and use a product kernel of the form:

$K_{h}(u)=\frac{1}{h^{d_{u}}} K\left(\frac{u_{1}}{h}, \ldots, \frac{u_{d_{u}}}{h}\right)$ with $K\left(u_{1}, \ldots, u_{d_{u}}\right)=\prod_{\ell=1}^{d_{u}} k\left(u_{\ell}\right)$,

where $d_{u}$ is the dimension of $u$ and $h \equiv h_{n}$ is the bandwidth that depends on $n$.

$\hat{\Delta}_{n, h}(\gamma)$ is an empirical version of $\Delta(\gamma)$. For each $\gamma \in \Gamma, \hat{\Delta}_{n, h}(\gamma)$ is a second order U-statistic. When $\hat{\Delta}_{n, h}(\gamma)$ is regarded as a process indexed by $\gamma \in \Gamma$, $\hat{\Delta}_{n, h}(\gamma)$ is a U-process. Note that $\left[\varphi\left(\tilde{W}_{i}^{\prime} \gamma\right)-\varphi\left(\tilde{W}_{i, j}^{\prime} \gamma\right)\right] K_{h}\left(Z_{i}-Z_{j}\right)$ is not symmetric in $i$ and $j$. To achieve the symmetry so that the theory of U-statistics and U-processes can be applied, we rewrite $\hat{\Delta}_{n, h}(\gamma)$ as

$\hat{\Delta}_{n, h}(\gamma)=\left(\begin{array}{l}n \\ 2\end{array}\right)^{-1} \sum_{i<j} \kappa_{h, 2}\left(W_{i}, W_{j} ; \gamma\right)$

where

$$
\begin{aligned}
\kappa_{h, 2}\left(W_{i}, W_{j} ; \gamma\right)= & \frac{1}{2}\left[\varphi\left(\tilde{W}_{i}^{\prime} \gamma\right)-\varphi\left(\tilde{W}_{i, j}^{\prime} \gamma\right)\right] K_{h}\left(Z_{i}-Z_{j}\right) \\
& +\frac{1}{2}\left[\varphi\left(\tilde{W}_{j}^{\prime} \gamma\right)-\varphi\left(\tilde{W}_{j, i}^{\prime} \gamma\right)\right] K_{h}\left(Z_{j}-Z_{i}\right)=\kappa_{h, 2}\left(W_{j}, W_{i} ; \gamma\right) .
\end{aligned}
$$

Our test statistic is related to what DG (2001, Sect. 5.2) propose but no formal proof is given there. DG formulate the null hypothesis as

$H_{0}: L\left(\gamma_{1}, \gamma_{2}, \gamma_{3}\right)=0$

for all $\left(\gamma_{1}^{\prime}, \gamma_{2}^{\prime}, \gamma_{3}^{\prime}\right)^{\prime}$ in the support of $\left(X^{\prime}, Y^{\prime}, Z^{\prime}\right)^{\prime}$ where

$L\left(\gamma_{1}, \gamma_{2}, \gamma_{3}\right)=E\left\{\left[1_{\gamma_{2}}(Y)-E\left(1_{\gamma_{2}}(Y) \mid Z\right)\right] 1_{\gamma_{1}}(X) 1_{\gamma_{3}}(Z) f_{Z}(Z)\right\}$

and $1_{v}(V)=1\{V \leq v\}$ is the indicator function. The DG statistic is based on the following estimator of $L\left(\gamma_{1}, \gamma_{2}, \gamma_{3}\right)$ :

$\hat{L}_{n, h}\left(\gamma_{1}, \gamma_{2}, \gamma_{3}\right)$

$$
=\frac{1}{n(n-1)} \sum_{i=1}^{n} \sum_{j=1, j \neq i}^{n}\left[1_{\gamma_{2}}\left(Y_{i}\right)-1_{\gamma_{2}}\left(Y_{j}\right)\right] 1_{\gamma_{1}}\left(X_{i}\right) 1_{\gamma_{3}}\left(Z_{i}\right) K_{h}\left(Z_{j}-Z_{i}\right)
$$




$$
\begin{aligned}
=\frac{1}{n(n-1)} \sum_{i=1}^{n} \sum_{j=1, j \neq i}^{n}\{ & {\left[1_{\gamma_{1}}\left(X_{i}\right) 1_{\gamma_{2}}\left(Y_{i}\right) 1_{\gamma_{3}}\left(Z_{i}\right)-1_{\gamma_{1}}\left(X_{i}\right) 1_{\gamma_{2}}\left(Y_{j}\right) 1_{\gamma_{3}}\left(Z_{i}\right)\right] } \\
\times & \left.K_{h}\left(Z_{j}-Z_{i}\right)\right\} .
\end{aligned}
$$

Comparing this with $\hat{\Delta}_{n, h}(\gamma)$ given in (15), we can see that $\hat{L}_{n, h}(\gamma)$ takes the same form as $\hat{\Delta}_{n, h}(\gamma)$.

The difference is that we use a GCR function $\varphi(x, y, z ; \gamma)$ while DG use the indicator function $1_{\gamma_{1}}(x) 1_{\gamma_{2}}(y) 1_{\gamma_{3}}(z)$. This has both theoretical and practical implications. First, from a theoretical point of view, while the indicator function is comprehensively revealing, it is not generically and comprehensively revealing. An advantage of using a GCR function is that the alternative hypothesis can be revealed by essentially all $\gamma$. This property does not hold for the indicator function, i.e., there may exist a region of $\left(\gamma_{1}, \gamma_{2}, \gamma_{3}\right)$ with nonempty interior such that the moment condition in (17) holds but $Y \not\lfloor X \mid Z$. Second, the GCR approach requires bounded random variables while the DG approach does not. So in our setting, the boundary smoothing bias cannot be avoided and has to be dealt with explicitly and rigorously. DG assume unbounded supports and so they do not have to deal with the boundary problem. Third, there can be a practical problem when computing some functionals of $\hat{L}_{n, h}\left(\gamma_{1}, \gamma_{2}, \gamma_{3}\right)$. For instance, the Cramér-von Mises statistic in DG (2001) takes the form

$C_{n}=\sum_{i=1}^{n} \hat{L}_{n, h}^{2}\left(X_{i}, Y_{i}, Z_{i}\right)$

where by definition

$$
\begin{aligned}
\hat{L}_{n, h}^{2}\left(X_{i}, Y_{i}, Z_{i}\right)=\frac{1}{n(n-1)} \sum_{k=1}^{n} \sum_{j=1, j \neq k}^{n}\{ & {\left[1_{Y_{i}}\left(Y_{k}\right)-1_{Y_{i}}\left(Y_{j}\right)\right] } \\
& \left.\times 1_{X_{i}}\left(X_{k}\right) 1_{Z_{i}}\left(Z_{k}\right) K_{h}\left(Z_{j}-Z_{k}\right)\right\} .
\end{aligned}
$$

The DG test rejects the null when $C_{n}$ is larger than an asymptotically valid critical value. When the dimensions of $X$ and $Z$ are large, $X_{k} \leq X_{i}$ and $Z_{k} \leq Z_{i}$ for $k \neq i$ may never happen and $1_{X_{i}}\left(X_{k}\right) 1_{Z_{i}}\left(Z_{k}\right)$ may never be different from zero, even in large samples. In this case $\hat{L}_{n, h}^{2}\left(X_{i}, Y_{i}, Z_{i}\right)$ is zero. This could have adverse effects on the size accuracy and the power property of the test in finite samples. This is supported by our Monte Carlo study.

A desirable property of the DG test is that it is invariant to strictly monotonic transformations of $\left\{X_{i}\right\}$ and $\left\{Y_{i}\right\}$. To see this, let $m_{X}(X)=\left(m_{X_{1}}\left(X_{1}\right), \ldots\right.$, $\left.m_{X_{d_{X}}}\left(X_{d_{X}}\right)\right)^{\prime}$ and $m_{Y}(Y)=\left(m_{Y_{1}}\left(Y_{1}\right), \ldots, m_{Y_{d_{Y}}}\left(Y_{d_{Y}}\right)\right)^{\prime}$ where all the univariate functions $m_{X_{k}}(\cdot)$ and $m_{Y_{k}}(\cdot)$ are strictly monotonic. Then

$$
\begin{aligned}
& \quad\left[1_{Y_{i}}\left(Y_{k}\right)-1_{Y_{i}}\left(Y_{j}\right)\right] 1_{X_{i}}\left(X_{k}\right) \\
& \quad=\left[1_{m_{Y}\left(Y_{i}\right)}\left(m_{Y}\left(Y_{k}\right)\right)-1_{m_{Y}\left(Y_{i}\right)}\left(m_{Y}\left(Y_{j}\right)\right)\right] 1_{m_{X}\left(X_{i}\right)}\left(m_{X}\left(X_{k}\right)\right) .
\end{aligned}
$$


As a result, $\hat{L}_{n, h}^{2}\left(X_{i}, Y_{i}, Z_{i}\right)$ is invariant to strictly monotonic transformations of $\left\{X_{i}\right\}$ and $\left\{Y_{i}\right\}$. This is an appealing property that is not shared by the GCR test.

More broadly, one may argue that a conditional independence test should be invariant to measurable and invertible transformations of all the variables $\left\{X_{i}\right\},\left\{Y_{i}\right\}$, and $\left\{Z_{i}\right\}$. The DG test is not invariant under this stronger notion of invariance. However, tests based on nonparametric estimators of some divergence measures between probability distributions such as Shannon's entropy metric or Hellinger's distance measure may be invariant in the stronger sense. An example of such a test is SW (2008) which is based on a weighted Hellinger distance between $f_{X Y Z}(x, y, z) f_{Z}(z)$ and $f_{X Z}(x, z) f_{Y Z}(y, z)$. Like the GCR test, the omnibus tests of LG (1997) and SW (2007) are not invariant to measurable and invertible transformations or strictly monotonic transformations.

While transformation invariance is a pleasant property, the invariance requirement is often invoked to reduce the class of tests under consideration so that uniformly most powerful invariance tests (UMPI) can be designed. However, in the present context, without specifying the direction of local alternatives, there is no uniformly most powerful test even among the class of invariance tests. We avoid choosing a direction in order to hedge against the possibility of having no power in other directions. The lack of a UMPI test makes the invariance requirement not as compelling as in some other settings where a UMPI test exists.

We view the GCR test and other tests, invariant or not, as complementary. For example, while the SW (2008) test, which is invariant, can be powerful in detecting high-frequency alternatives, it suffers from the curse of dimensionality, as discussed above. In contrast, the GCR test, which is not invariant, does not suffer from the curse of dimensionality and is more powerful against low-frequency departures. In addition, the GCR test may be made invariant to strictly monotonic transformations if we convert the data $\left\{X_{i}\right\}$ and $\left\{Y_{i}\right\}$ to ranks before applying the GCR test. A systematic study of this rank-based GCR test is beyond the scope of this paper.

\section{STOCHASTIC APPROXIMATIONS AND FINITE DIMENSIONAL CONVERGENCE}

\subsection{Assumptions}

In this subsection, we state the assumptions that are required to establish the asymptotic properties of $\hat{\Delta}_{n, h}(\gamma)$. We start with a definition, which uses the following multi-index notation: for $j=\left(j_{1}, \ldots, j_{m}\right)$ with $j_{\ell}$ being nonnegative integers, we denote $|j|=j_{1}+j_{2}+\cdots+j_{m}, j !=j_{1} ! \cdots j_{m} !, u^{j}=u_{1}^{j} \cdots u_{m}^{j_{m}}$, and $D^{j} g(u)=\partial^{|j|} g(u) / \partial u_{1}^{j_{1}} \cdots \partial u_{m}^{j_{m}}$.

DEFINITION 2. $\mathcal{G}_{\beta}(\mathcal{A}, \epsilon, \rho, m), \beta>1$, is a class of functions $g_{\alpha}(\cdot): \mathbb{R}^{m} \rightarrow \mathbb{R}$ indexed by $\alpha \in \mathcal{A}$ satisfying the following two conditions: 
(a) for each $\alpha, g_{\alpha}(\cdot)$ is $b$ times continuously differentiable, where $b$ is the greatest integer that is smaller than $\beta$;

(b) let $Q_{\alpha}(u, v)$ be the Taylor series expansion of $g_{\alpha}(u)$ around $v$ of order $b$ :

$$
Q_{\alpha}(u, v)=\sum_{j:|j| \leq b, j \neq 0} \frac{D^{j} g_{\alpha}(v)}{j !}(u-v)^{j}
$$

then

$$
\sup _{\alpha \in \mathcal{A}\|u-v\| \leq \epsilon} \sup _{\left\|g_{\alpha}(u)-g_{\alpha}(v)-Q_{\alpha}(u, v)\right\|} \leq \rho
$$

for some constants $\epsilon>0$ and $\rho>0$.

In the absence of the index set $\mathcal{A}$, we use $\mathcal{G}_{\beta}(\epsilon, \rho, m)$ to denote the class of functions. In this case, our definition is similar to Definition 2 in Robinson (1988) and Definition 2 in DG (2001). A sufficient condition for condition (b) is that the partial derivative of the $b$-th order is uniformly Hölder continuous:

$\sup _{\alpha \in \mathcal{A}\|v-u\| \leq \epsilon} \sup _{\|}\left|D^{j} g_{\alpha}(u)-D^{j} g_{\alpha}(v)\right| \leq \epsilon^{\beta-b}$

for all $j$ such that $|j|=b$.

We are ready to present our assumptions.

Assumption 1 (IID). ( $a)\left\{W_{i} \in[0,1]^{d}\right\}_{i=1}^{n}$ is an IID sequence of random variables on the complete probability space $(\Omega, \mathcal{F}, \mathcal{P}) ;(b)$ each element $Z_{\ell}$ of $Z$ is supported on $[0,1] ;(c)$ the distribution of $Z$ admits a density function $f_{Z}(z)$ with respect to the Lebesgue measure.

Assumption 2 (Smoothness of the Densities). (a) $f_{Z}(\cdot) \in \mathcal{G}_{q+1}\left(\epsilon, \rho, d_{Z}\right.$ ) for some integer $q>0$ and some constants $\epsilon>0$ and $\rho>0$; $(b) D^{j} f_{Z}(\breve{z})=0$ for all $0 \leq|j| \leq q$ and all $\breve{z}$ on the boundary of $[0,1]^{d Z} ;(c)$ the conditional distribution functions $F_{Y \mid Z}, F_{X \mid Z}$, and $F_{X Y \mid Z}$ admit the respective densities $f_{Y \mid Z}(y \mid z), f_{X \mid Z}(x \mid z)$, and $f_{X Y \mid Z}(x, y \mid z)$ with respect to a finite counting measure, or the Lebesgue measure or their product measure; $(d)$ as functions of $z$ indexed by $x, y$, or $(x, y) \in \mathcal{A}, f_{X \mid Z}(x \mid z), f_{Y \mid Z}(y \mid z)$, and $f_{X Y \mid Z}(x \mid z)$ belong to $\mathcal{G}_{q+1}\left(\mathcal{A}, \epsilon, \rho, d_{Z}\right)$ with $\mathcal{A}=[0,1]^{d_{X}},[0,1]^{d_{Y}}$, or $[0,1]^{d_{X}+d_{Y}}$.

Assumption 3 (GCR). (a) $\Gamma$ is compact with nonempty interior; $(b) \varphi \in$ $\mathcal{G}_{\beta}(\epsilon, \rho, 1)$.

Assumption 4 (Kernel Function). The univariate kernel $k(\cdot)$ is the $q$ th order symmetric and bounded kernel $k: \mathbb{R} \rightarrow \mathbb{R}$ such that

(a) $\int k(v) d v=1, \int v^{j} k(v) d v=0$ for $j=1,2, \ldots, q-1$;

(b) $k(v)=O\left(\left(1+|v|^{\xi}\right)^{-1}\right)$ for some $\xi>q^{2}+q+2$. 
Assumption 5 (Bandwidth). The bandwidth $h=h_{n}$ satisfies

(a) $n h^{d_{Z}} \rightarrow \infty$ as $n \rightarrow \infty$;

(b) $\sqrt{n} h^{q}=o(1)$, i.e., $h=o\left(n^{-1 /(2 q)}\right)$ as $n \rightarrow \infty$.

Some discussions on the assumptions are in order. The IID condition in Assumption 1 is maintained for convenience. Analogous results hold under weaker conditions, but we leave explicit consideration of these aside.

Assumptions 2(a) and (d) are needed to control the smoothing bias. Under Assumptions 1 (b) and 2(a), we have $\int f_{Z}^{2}(z) d z<\infty$. So it is not necessary to state the square integrability of $f_{Z}(z)$ as a separate assumption. In assumption 2(d), the smoothness condition is with respect to the conditioning variable $Z$. It does not require the marginal distributions of $X$ and $Y$ to be smooth. In fact, $X$ and $Y$ could be either discrete or continuous. In addition, from a technical point of view, we only need to assume that there exists a version of the conditional density functions satisfying Assumption 2(d).

Assumption 2(b) is a technical condition, which helps avoid the boundary bias problem, a well-known problem for density estimation at the boundary. The GCR approach of StW requires the boundedness of the random vectors, so we have to deal with the boundary bias problem. If Assumption 2(b) does not hold, we can transform $Z$ into $Z_{\Theta}=\left(\Theta^{-1}\left(Z_{1}\right), \Theta^{-1}\left(Z_{2}\right), \ldots, \Theta^{-1}\left(Z_{d_{Z}}\right)\right)^{\prime}$, where $\Theta:[0,1] \rightarrow[0,1]$ is strictly increasing and $q+1$ times continuously differentiable with inverse $\Theta^{-1}$. Now

$$
\begin{aligned}
P\left\{Z_{\Theta}<z\right\} & =P\left\{Z_{1}<\Theta\left(z_{1}\right), \ldots, Z_{d_{Z}}<\Theta\left(z_{d_{Z}}\right)\right\} \\
& =F_{Z}\left(\Theta\left(z_{1}\right), \ldots, \Theta\left(z_{d_{Z}}\right)\right),
\end{aligned}
$$

and the density of $Z_{\Theta}$ is $f_{Z_{\Theta}}(z)=f_{Z}(\Theta(z)) \Theta^{\prime}\left(z_{1}\right) \ldots \Theta^{\prime}\left(z_{d_{Z}}\right)$. So if $\Theta^{(i)}(0)=$ $\Theta^{(i)}(1)=0$ for $i=0, \ldots, q$, then Assumption 2(b) is satisfied for the transformed random vector $Z_{\Theta}$ and we can work with $Z_{\Theta}$ rather than $Z$. We can do so because $Y \perp X \mid Z$ if and only if $Y \perp X \mid Z_{\Theta}$. An example of $\Theta$ is the CDF of a beta distribution:

$\Theta(v)=\frac{1}{B(q+1, q+1)} \int_{0}^{v} x^{q}(1-x)^{q} d x:=\frac{\mathbb{B}(v, q+1, q+1)}{\mathbb{B}(1, q+1, q+1)}$,

where $\mathbb{B}(v, q+1, q+1)=\int_{0}^{v} x^{q}(1-x)^{q} d x$ is the incomplete beta function.

The idea of using transformations to remove the boundary bias has a long history; see Geenens (2014) and the references therein. However, our idea is different here. In Geenens (2014) and the related literature, in order to reduce the boundary bias, a transformation is employed to map a bounded support into an unbounded support. This approach clearly is not compatible with the GCR framework we use here. Our idea is to transform the data so that the probability mass around the boundary is relatively small. This is a viable approach as long as our focus is not the pdf of the original data per se. This idea may be of independent interest. 
Another often used approach to handle the boundary problem is to trim the data when $Z$ is boundedly supported. Let $\mathcal{Z}_{\varepsilon}$ be a proper subset of the support of $Z$ satisfying $P\left[Z_{i} \in \mathcal{Z}_{\varepsilon}\right]=1-\varepsilon$ for some $\varepsilon$ approaching zero at a certain rate. In this approach, we replace $K_{h}\left(Z_{i}-Z_{j}\right)$ in the definition of $\hat{\Delta}_{n, h}(\gamma)$ by $K_{h}\left(Z_{i}-Z_{j}\right)$. $1\left[Z_{i} \in Z_{\varepsilon}\right] \cdot 1\left[Z_{j} \in Z_{\varepsilon}\right]$. For example, this approach is used in $\mathrm{Li}$ and Fan $(2003$, p. 745) in a different context. Note that the use of transformation or trimming is more of theoretical importance, as they are necessary to achieve the parametric $\sqrt{n}$ rate of convergence of $\hat{\Delta}_{n, h}(\gamma)$ to $\Delta(\gamma)$. In practice, transformation and trimming may or may not be important. When the probability mass near the boundary is relatively small, they are likely to be unimportant. In this case, we may skip the transformation or trimming and ignore the boundary bias in practice.

Assumption 3(a) is needed only when we attempt to establish the uniformity of some asymptotic properties over $\Gamma$. Like Assumption 2, Assumption 3(b) helps control the smoothing bias. It is satisfied by many GCR functions such as exp $(\cdot)$, normal PDF, $\sin (\cdot)$, and $\cos (\cdot)$.

The conditions on the high order kernel in Assumption 4 are fairly standard. For example, both Robinson (1988) and DG (2001) make a similar assumption. The only difference is that Robinson (1988) and DG (2001) require that $\xi>q+1$, while we require a stronger condition that $\xi>q^{2}+q+2$ in Assumption 4(b). The stronger condition is needed to control the boundary bias, which is absent in Robinson (1988) and DG (2001), as they assume that $Z$ has an unbounded support. Assumption 4(b) is not restrictive. It is satisfied by typical kernels used in practice, as they are either supported on $[0,1]$ or have exponentially decaying tails.

Assumption 5(a) ensures that the degenerate U-statistic in the Hoeffding decomposition of $\hat{\Delta}_{n, h}(\gamma)$ is asymptotically negligible. Assumption 5(b) removes the dominating bias of $\hat{\Delta}_{n, h}(\gamma)$. See Lemmas 3 and 4 below. A necessary condition for Assumption 5 to hold is that $2 q>d_{Z}$.

\subsection{Stochastic Approximations}

To establish the asymptotic properties of $\hat{\Delta}_{n, h}(\gamma)$, we develop some stochastic approximations, using the theory of U-statistics and U-processes pioneered by Hoeffding (1948).

Let $\kappa_{h, 1}(w ; \gamma)=E \kappa_{h, 2}\left(w, W_{j} ; \gamma\right)$. Using Hoeffding's H-decomposition, we can decompose $\hat{\Delta}_{n, h}(\gamma)$ as

$$
\hat{\Delta}_{n, h}(\gamma)=\Delta_{h}(\gamma)+H_{n, h}(\gamma)+R_{n, h}(\gamma),
$$

where

$$
\begin{aligned}
\Delta_{h}(\gamma) & =E \kappa_{h, 2}\left(W_{j}, W_{i} ; \gamma\right)=E \kappa_{h, 1}\left(W_{i} ; \gamma\right) \\
H_{n, h}(\gamma) & =\frac{2}{n} \sum_{i=1}^{n} \tilde{\kappa}_{h, 1}\left(W_{i} ; \gamma\right)
\end{aligned}
$$


$R_{n, h}(\gamma)=\left(\begin{array}{l}n \\ 2\end{array}\right)^{-1} \sum_{i<j} \tilde{\kappa}_{h, 2}\left(W_{i}, W_{j}, \gamma\right)$

and

$$
\begin{aligned}
\tilde{\kappa}_{h, 1}\left(W_{i} ; \gamma\right) & =\kappa_{h, 1}\left(W_{i} ; \gamma\right)-\Delta_{h}(\gamma) \\
\tilde{\kappa}_{h, 2}\left(W_{i}, W_{j}, \gamma\right) & =\kappa_{h, 2}\left(W_{i}, W_{j} ; \gamma\right)-\kappa_{h, 1}\left(W_{i} ; \gamma\right)-\kappa_{h, 1}\left(W_{j} ; \gamma\right)+\Delta_{h}(\gamma) .
\end{aligned}
$$

The sum of the first two terms in the H-decomposition is known as the Hájek projection. For easy reference, we denote it as

$$
\tilde{\Delta}_{n, h}(\gamma)=\Delta_{h}(\gamma)+H_{n, h}(\gamma)
$$

By construction, $H_{n, h}(\gamma)$ and $R_{n, h}(\gamma)$ are uncorrelated zero mean random variables. We show that the projection remainder $R_{n, h}(\gamma)$ is asymptotically negligible, and as a result $\hat{\Delta}_{n, h}(\gamma)$ and its Hájek projection $\tilde{\Delta}_{n, h}(\gamma)$ have the same limiting distribution.

For each given $\gamma$ and $h, R_{n, h}(\gamma)$ is a degenerate second order U-statistic with kernel $\tilde{\kappa}_{h, 2}(\cdot, \cdot ; \gamma)$. According to the theory of U-statistics (e.g., Lee, 1990), we have

$$
\operatorname{var}\left[R_{n, h}(\gamma)\right]=\frac{2}{n(n-1)} \operatorname{var}\left[\tilde{\kappa}_{h, 2}\left(W_{i}, W_{j}, \gamma\right)\right]
$$

This can also be proved directly by observing that $\tilde{\kappa}_{h, 2}\left(W_{i}, W_{j}, \gamma\right)$ is uncorrelated with $\tilde{\kappa}_{h, 2}\left(W_{\ell}, W_{m}, \gamma\right)$ if $(i, j) \neq(\ell, m)$.

If $h$ were fixed, then it follows from the basic U-statistic theory that $R_{n, h}(\gamma)=$ $o_{p}(1 / \sqrt{n})$ for each $\gamma \in \Gamma$. However, in the present setting, $h \rightarrow 0$ as $n \rightarrow \infty$, so the basic U-statistic theory does not directly apply. Nevertheless, we can still show that $R_{n, h}(\gamma)$ is still $o_{p}\left(n^{-1 / 2}\right)$ under Assumption 5(a). In fact, we can prove a stronger result, as Lemma 3 shows.

LEMMA 3. Under Assumptions 1-5(a), if $h \rightarrow 0$ as $n \rightarrow \infty$, then $\sup _{\gamma \in \Gamma} \sqrt{n} R_{n, h}(\gamma)=o_{p}(1)$.

We proceed to establish a stochastic approximation of the Hájek projection $\tilde{\Delta}_{n, h}(\gamma)$. Note that both $\Delta_{h}(\gamma)$ and $H_{n, h}(\gamma)$ depend on $h$. Using a Taylor expansion, we can separate terms independent of $h$ from those associated with $h$ in $\Delta_{h}(\gamma)$ and $H_{n, h}(\gamma)$. By using a higher order kernel $K$ and controlling the rate of $h$ so that it shrinks fast enough, we can ensure that the terms associated with $h$ vanish asymptotically, as in Powell, Stock, and Stoker (1989).

More specifically, we first show that $\Delta_{h}(\gamma)=\Delta(\gamma)+O\left(h^{q}\right)$, where $q$ is the order of the kernel $k$. Then we show that $H_{n, h}(\gamma)=2 n^{-1} \sum_{i=1}^{n}$ $\left\{\kappa_{1}\left(W_{i} ; \gamma\right)-E\left[\kappa_{1}\left(W_{i} ; \gamma\right)\right]\right\}+O_{p}\left(h^{q}\right)$, where 


$$
\begin{aligned}
\kappa_{1}\left(W_{i} ; \gamma\right) \equiv & \frac{1}{2} \varphi\left(\gamma_{0}+X_{i}^{\prime} \gamma_{1}+Y_{i}^{\prime} \gamma_{2}+Z_{i}^{\prime} \gamma_{3}\right) f_{Z}\left(Z_{i}\right) \\
& -\frac{1}{2} \int \varphi\left(\gamma_{0}+X_{i}^{\prime} \gamma_{1}+y^{\prime} \gamma_{2}+Z_{i}^{\prime} \gamma_{3}\right) f_{Y Z}\left(y, Z_{i}\right) d y \\
& +\frac{1}{2} \int \varphi\left(\gamma_{0}+x^{\prime} \gamma_{1}+y^{\prime} \gamma_{2}+Z_{i}^{\prime} \gamma_{3}\right) f_{X Y Z}\left(x, y, Z_{i}\right) d x d y \\
& -\frac{1}{2} \int \varphi\left(\gamma_{0}+x^{\prime} \gamma_{1}+Y_{i}^{\prime} \gamma_{2}+Z_{i}^{\prime} \gamma_{3}\right) f_{X Z}\left(x, Z_{i}\right) d x
\end{aligned}
$$

Under Assumption 5(b), $\sqrt{n} h^{q} \rightarrow 0$, which makes both the second term of $\Delta_{h}(\gamma)$ and the second term of $H_{n, h}(\gamma)$ vanish asymptotically. The following lemma presents these results formally.

LEMMA 4. Let Assumptions 1-4 and 5(b) hold. Then

(a) $\sqrt{n}\left[\Delta_{h}(\gamma)-\Delta(\gamma)\right]=o$ (1) uniformly over $\gamma \in \Gamma$;

(b) $\sqrt{n} H_{n, h}(\gamma)=2 / \sqrt{n} \sum_{i=1}^{n}\left\{\kappa_{1}\left(W_{i} ; \gamma\right)-E\left[\kappa_{1}\left(W_{i} ; \gamma\right)\right]\right\}+o_{p}(1)$ uniformly over $\gamma \in \Gamma$.

It follows from Lemmas 3 and 4 that

$$
\begin{aligned}
& \sqrt{n}\left[\hat{\Delta}_{n, h}(\gamma)-\Delta(\gamma)\right] \\
& \quad=\sqrt{n} H_{n, h}(\gamma)+\sqrt{n} R_{n, h}(\gamma)+\sqrt{n}\left[\Delta_{h}(\gamma)-\Delta(\gamma)\right] \\
& \quad=\sqrt{n} H_{n, h}(\gamma)+o_{p}(1)=\frac{2}{\sqrt{n}} \sum_{i=1}^{n}\left\{\kappa_{1}\left(W_{i} ; \gamma\right)-E\left[\kappa_{1}\left(W_{i} ; \gamma\right)\right]\right\}+o_{p}(1)
\end{aligned}
$$

uniformly over $\gamma \in \Gamma$. So $\sqrt{n}\left[\hat{\Delta}_{n, h}(\gamma)-\Delta(\gamma)\right]$ and $2 / \sqrt{n} \sum_{i=1}^{n}$ $\left\{\kappa_{1}\left(W_{i} ; \gamma\right)-E\left[\kappa_{1}\left(W_{i} ; \gamma\right)\right]\right\}$ have the same limiting distribution for each $\gamma \in \Gamma$.

\subsection{Finite Dimensional Convergence}

In this subsection, we view $\hat{\Delta}_{n, h}(\gamma)$ as a U-process indexed by $\gamma$ and consider its finite-dimensional convergence.

Let $\Gamma_{s}=\left\{\gamma_{1}, \gamma_{2}, \ldots, \gamma_{s}\right\}$ for some $s<\infty$ and $\gamma_{\ell} \in \Gamma$, and define

$\hat{\Delta}_{n, h}\left(\Gamma_{s}\right):=\left[\hat{\Delta}_{n, h}\left(\gamma_{1}\right), \hat{\Delta}_{n, h}\left(\gamma_{2}\right), \ldots, \hat{\Delta}_{n, h}\left(\gamma_{s}\right)\right]^{\prime}$.

Similarly, we define $\Delta\left(\Gamma_{s}\right):=\left[\Delta\left(\gamma_{1}\right), \Delta\left(\gamma_{2}\right), \ldots, \Delta\left(\gamma_{s}\right)\right]^{\prime}$. Theorem 5 below establishes the asymptotic normality of $\sqrt{n}\left[\hat{\Delta}_{n, h}\left(\Gamma_{s}\right)-\Delta\left(\Gamma_{s}\right)\right]$.

THEOREM 5. Let Assumptions 1-5 hold. Then

$\sqrt{n}\left[\hat{\Delta}_{n, h}\left(\Gamma_{s}\right)-\Delta\left(\Gamma_{s}\right)\right] \stackrel{d}{\rightarrow} N(0, \Omega)$, 
where the $(\ell, m)$ element of $\Omega$ is

$\Omega(\ell, m):=\sigma_{\Delta}\left(\gamma_{\ell}, \gamma_{m}\right)=4 \operatorname{cov}\left[\kappa_{1}\left(W_{i} ; \gamma_{\ell}\right), \kappa_{1}\left(W_{i} ; \gamma_{m}\right)\right]$

If, in addition, $H_{0}$ holds, then $\Delta(\gamma)=0$, and

$\sigma_{\Delta}\left(\gamma_{\ell}, \gamma_{m}\right)=4 E\left[\Lambda\left(W_{i} ; \gamma_{\ell}\right) \Lambda\left(W_{i} ; \gamma_{m}\right)\right]$

where

$$
\begin{aligned}
\Lambda\left(W_{i} ; \gamma\right)= & \frac{1}{2} E\left[\varphi\left(\tilde{W}_{i}^{\prime} \gamma\right) f_{Z}\left(Z_{i}\right) \mid X_{i}, Y_{i}, Z_{i}\right]-\frac{1}{2} E\left[\varphi\left(\tilde{W}_{i}^{\prime} \gamma\right) f_{Z}\left(Z_{i}\right) \mid X_{i}, Z_{i}\right] \\
& -\frac{1}{2} E\left[\varphi\left(\tilde{W}_{i}^{\prime} \gamma\right) f_{Z}\left(Z_{i}\right) \mid Y_{i}, Z_{i}\right]+\frac{1}{2} E\left[\varphi\left(\tilde{W}_{i}^{\prime} \gamma\right) f_{Z}\left(Z_{i}\right) \mid Z_{i}\right] .
\end{aligned}
$$

Theorem 5 is of interest in its own right. For example, we can use it to construct a Wald test. There may be some power loss if $s$ is small. When $s$ is large enough such that $\Gamma_{s}$ approximates $\Gamma$ very well, then the power loss will be small. The idea can be motivated from the method of sieves. We do not pursue this here but refer to Huang (2009) for more discussions. Instead, we consider the ICM tests in Section 4. Theorem 5 is an important first step in obtaining the asymptotic distributions of the ICM statistics.

Observe that $\hat{\Delta}_{n, h}(\gamma)$ is not symmetric in $X$ and $Y$, whereas the hypothesis $Y \perp X \mid Z$ is. However, $\sqrt{n}\left[\hat{\Delta}_{n, h}(\gamma)-\Delta_{h}(\gamma)\right]$ is asymptotically equivalent to $2 / \sqrt{n} \sum_{i=1}^{n}\left[\kappa_{1}\left(W_{i} ; \gamma\right)-E \kappa_{1}\left(W_{i} ; \gamma\right)\right]$. It can be readily checked that $\kappa_{1}(W ; \gamma)$ is symmetric in $Y$ and $X$. Alternatively, we can follow the definition of $g_{X Z}$ in (14) and define $g_{Y Z}(y, z ; \gamma), g_{Z}(z ; \gamma)$, and $g_{X Y Z}(x, y, z ; \gamma)$ as

$$
\begin{aligned}
g_{Y Z}(y, z ; \gamma) & =E[\varphi(X, y, z ; \gamma) \mid Z=z] \\
g_{Z}(z ; \gamma) & =E[\varphi(X, Y, z ; \gamma) \mid Z=z] \\
g_{X Y Z}(x, y, z ; \gamma) & =E[\varphi(x, y, z ; \gamma) \mid Z=z]=\varphi(x, y, z ; \gamma),
\end{aligned}
$$

where the last equality is tautological. Then

$$
\begin{aligned}
\kappa_{1}(W ; \gamma)=\frac{1}{2}[ & g_{X Y Z}(X, Y, Z ; \gamma)-g_{X Z}(X, Z ; \gamma) \\
& \left.-g_{Y Z}(Y, Z ; \gamma)+g_{Z}(Z ; \gamma)\right] f_{Z}(Z),
\end{aligned}
$$

which is clearly symmetric in $Y$ and $X$. If we construct another estimator, say $\hat{\Delta}_{n, h}^{*}(\gamma)$, by switching the roles of $X$ and $Y$, we can show that $\hat{\Delta}_{n, h}^{*}$ and $\hat{\Delta}_{n, h}(\gamma)$ are asymptotically equivalent in the sense that $\sqrt{n}\left[\hat{\Delta}_{n, h}^{*}-\hat{\Delta}_{n, h}(\gamma)\right]=o_{p}(1)$ uniformly over $\gamma \in \Gamma$. So there is no asymptotic gain in taking an average of $\hat{\Delta}_{n, h}(\gamma)$ and $\hat{\Delta}_{n, h}^{*}$. This point is further supported by the symmetry of $\Lambda(W ; \gamma)$ in $X$ and $Y$. 


\subsection{Bandwidth Selection}

Although any choice of bandwidth $h$ satisfying Assumption 5 will deliver the asymptotic distribution in Theorem 5 , in practice we need some guidance on how to select $h$. Ideally we should select an $h$ that would give us the greatest power for a given size of test, but deriving that procedure would be complicated enough to justify another study. Moreover, it would only make a difference for higher order results. Thus, for the present purposes, we just provide a simple "plugin" estimator of the MSE-minimizing bandwidth proposed by Powell and Stoker (1996).

Since the test statistic is based on $\hat{\Delta}_{n, h}(\gamma)$, which estimates $\Delta(\gamma)$, it is appealing to choose an $h$ that minimizes the mean squared error (MSE) of $\hat{\Delta}_{n, h}(\gamma)$. After some tedious but straightforward calculations, we get

$$
\begin{aligned}
\operatorname{MSE}\left[\hat{\Delta}_{n, h}(\gamma)\right]= & \left(\Delta_{h}(\gamma)-\Delta(\gamma)\right)^{2}+\operatorname{var}\left[\hat{\Delta}_{n, h}(\gamma)\right] \\
= & \left\{E\left[B_{5}(W ; \gamma)\right]\right\}^{2} h^{2 q}+o\left(h^{2 q}\right)+\operatorname{var}\left[\hat{\Delta}_{n, h}(\gamma)\right] \\
= & \left\{E\left[B_{5}(W ; \gamma)\right]\right\}^{2} h^{2 q}+o\left(h^{2 q}\right) \\
& +4 n^{-1} \operatorname{var}\left[\kappa_{1}(W ; \gamma)\right]+4 n^{-1} C_{0}(\gamma) h^{q}+o\left(n^{-1} h^{q}\right) \\
& -4 n^{-2} \operatorname{var}\left[\kappa_{1}(W ; \gamma)\right]+2 n^{-2} E[\delta(W ; \gamma)] h^{-d_{Z}} \\
& +o\left(n^{-2} h^{-d_{Z}}\right)-2 n^{-2} \Delta(\gamma)^{2}+o\left(n^{-2}\right),
\end{aligned}
$$

where $B_{5}$ is defined in (A.9) in the appendix, and $\delta(W ; \gamma)$ is defined by

$$
\begin{aligned}
E\left[\left\|\kappa_{h, 2}\left(W_{i}, W_{j}, \gamma\right)\right\|^{2} \mid W_{i}\right] & =\delta\left(W_{i} ; \gamma\right) h^{-d_{Z}}+\delta^{*}\left(W_{i}, h ; \gamma\right), \text { where } \\
E\left(\left\|\delta^{*}\left(W_{i}, h ; \gamma\right)\right\|\right) & =o\left(h^{-d_{Z}}\right) .
\end{aligned}
$$

The term $4 n^{-1} \operatorname{var}\left[\kappa_{1}(W ; \gamma)\right]-4 n^{-2} \operatorname{var}\left[\kappa_{1}(W ; \gamma)\right]$ does not depend on $h$. The term $2 n^{-2} \Delta(\gamma)^{2}$ must be of smaller order than $4 n^{-1} C_{0} h^{q}$, and $4 n^{-1} C_{0} h^{q}$ must be of smaller order than $\left\{E\left[B_{5}(W ; \gamma)\right]\right\}^{2} h^{2 q}$; otherwise there would be a contradiction to Assumption 5(b). So the leading term of $M S E\left[\hat{\Delta}_{n, h}(\gamma)\right]$ that involves $h$ is

$$
M S E_{1}\left[\hat{\Delta}_{n, h}(\gamma)\right]=\left\{E\left[B_{5}(W ; \gamma)\right]\right\}^{2} h^{2 q}+2 n^{-2} E[\delta(W ; \gamma)] h^{-d_{z}} .
$$

By minimizing $M S E_{1}\left[\hat{\Delta}_{n, h}(\gamma)\right]$, we obtain the optimal bandwidth

$$
h^{*}=\left[\frac{d_{Z} \cdot E[\delta(W ; \gamma)]}{q \cdot\left\{E\left[B_{5}(W ; \gamma)\right]\right\}^{2}}\right]^{1 /\left(2 q+d_{Z}\right)} \cdot\left[\frac{1}{n}\right]^{2 /\left(2 q+d_{Z}\right)} .
$$

Now Assumption 5(a) is satisfied:

$n\left(h^{*}\right)^{d_{Z}} \asymp n^{1-2 d_{Z} /\left(2 q+d_{Z}\right)} \asymp n^{\left(2 q-d_{Z}\right) /\left(2 q+d_{Z}\right)} \rightarrow \infty$, given $2 q>d_{Z}$.

And so is Assumption 5(b):

$\sqrt{n}\left(h^{*}\right)^{q} \asymp n^{1 / 2-2 q /\left(2 q+d_{Z}\right)} \asymp n^{-\left(2 q-d_{Z}\right) / 2\left(2 q+d_{Z}\right)}=o(1)$, given $2 q>d_{Z}$. 
The optimal bandwidth depends on the unknown quantities $E[\delta(W ; \gamma)]$ and $E\left[B_{5}(W ; \gamma)\right]$. Here we follow the standard practice (e.g., Powell and Stoker, 1996) and use a simple plug-in estimator of $h^{*}$. Let $h_{0}$ be an initial bandwidth. Suppose $E\left[\kappa_{h, 2}\left(W_{i}, W_{j} ; \gamma\right)^{4}\right]=O\left(h_{0}^{-\eta-2 d_{Z}}\right)$ for some $\eta>0$, and let $\varrho=\max \left\{\eta+2 d_{Z}, 2 q+d_{Z}\right\}$. If $h_{0} \rightarrow 0$ and $n h_{0}^{\varrho} \rightarrow \infty$, then by Powell and Stoker (1996, Prop. 4.2),

$\hat{\delta} \equiv \hat{\delta}\left(h_{0}\right)=\left(\begin{array}{l}n \\ 2\end{array}\right)^{-1} \sum_{i<j} h_{0}^{d_{Z}} \cdot\left[\kappa_{h_{0}, 2}\left(W_{i}, W_{j}, \gamma\right)\right]^{2} \stackrel{p}{\rightarrow} E\left[\delta\left(W_{i} ; \gamma\right)\right]$

and

$\hat{B}_{5} \equiv \frac{\hat{\Delta}_{n, \tau h_{0}}(\gamma)-\hat{\Delta}_{n, h_{0}}(\gamma)}{\left(\tau h_{0}\right)^{q}-h_{0}^{q}}$ for some $0<\tau \neq 1 \stackrel{p}{\rightarrow} E\left[B_{5}(W ; \gamma)\right]$.

The estimator $\hat{B}_{5}$ given above is a "slope" between two points $\left(h_{0}^{q}, \hat{\Delta}_{n, h_{0}}(\gamma)\right)$ and $\left(\tau h_{0}^{q}, \hat{\Delta}_{n, \tau h_{0}}(\gamma)\right)$. To get a more stable estimator, we could use a regression of $\hat{\Delta}_{n, h_{0}}(\gamma)$ on $h_{0}^{q}$ for various values of $h_{0}$. Given $\hat{\delta}$ and $\hat{B}_{5}$, the plug-in estimator of $h^{*}$ is

$\hat{h}=\left[\frac{d_{Z} \cdot \hat{\delta}}{q \cdot \hat{B}_{5}^{2}}\right]^{1 /\left(2 q+d_{Z}\right)} \cdot\left[\frac{1}{n}\right]^{2 /\left(2 q+d_{Z}\right)}$.

In practice we can choose $q$ large enough so that $\varrho=\max \left\{\eta+2 d_{Z}, 2 q+d_{Z}\right\}=$ $2 q+d_{Z}$; then we can choose the initial bandwidth to be $h_{0}=o\left(n^{-1 /\left(2 q+d_{Z}\right)}\right)$. The data driven $\hat{h}$ depends on $\gamma$. We may choose different bandwidths for different $\gamma$ 's. This is what we follow in our Monte Carlo experiments.

Powell and Stoker (1996) mention one technical proviso: $\hat{\Delta}_{n}(\gamma ; \hat{h})$ is not guaranteed to be asymptotically equivalent to $\hat{\Delta}_{n}\left(\gamma ; h^{*}\right)$ since the MSE calculations are based on the assumption that $h$ is deterministic. The suggested solution is to discretize the set of possible scaling constants, replacing $\hat{h}$ with the closest value, $\hat{h}^{\dagger}$, in some finite set. The estimation uncertainty in $\hat{h}^{\dagger}$ is small enough that it will not affect the asymptotic MSE.

\section{AN INTEGRATED CONDITIONAL MOMENT TEST}

In this section, we "integrate out" $\gamma$ to get an ICM type test statistic, following Bierens (1990) and StW (1998).

\subsection{The Test Statistic and its Asymptotic Null Distribution}

If $\varphi$ is GCR, testing $H_{0}: Y \perp X \mid Z$ is equivalent to testing $H_{0}: \Delta(\gamma)=0$ for essentially all $\gamma \in \Gamma$. In other words, if we view $\hat{\Delta}_{n, h}(\gamma)$ as a random function in $\gamma$, we are testing whether its mean function $\Delta(\gamma)$ is zero on $\Gamma$. If $\Gamma$ is compact, 
we can show that $\sqrt{n} \hat{\Delta}_{n, h}(\gamma)$ converges to a zero mean Gaussian process under the null. Based on $\sqrt{n} \hat{\Delta}_{n, h}(\gamma)$, we construct the ICM test statistic

$M_{n}=n \int_{\Gamma}\left[\hat{\Delta}_{n, h}(\gamma)\right]^{2} d \mu(\gamma)$,

where $\mu$ is a probability measure on $\Gamma$ that is absolutely continuous with respect to the Lebesgue measure on $\Gamma$. Here we integrate $\left[\hat{\Delta}_{n, h}(\gamma)\right]^{2}$, which gives a Cramer-von Mises (CM) type test. Alternatively, we could integrate $\left|\hat{\Delta}_{n, h}(\gamma)\right|^{p}$, $1 \leq p \leq \infty$. The choice $p=\infty$ (which gives the maximum over $\Gamma$ ) yields a Kolmogorov-Smirnov (KS) type test. We work with $p=2$ for concreteness and because CM-type tests often outperform KS-type tests.

To establish the weak convergence of $M_{n}$, we first show that $\sqrt{n}\left[\hat{\Delta}_{n, h}(\cdot)-\right.$ $\Delta(\cdot)$ ] converges to a Gaussian process. Define

$\zeta_{n}(\gamma)=\frac{2}{\sqrt{n}} \sum_{i=1}^{n}\left\{\kappa_{1}\left(W_{i} ; \gamma\right)-E\left[\kappa_{1}\left(W_{i} ; \gamma\right)\right]\right\}$.

Then Lemmas 3 and 4 imply that

$$
\sup _{\gamma \in \Gamma}\left|\sqrt{n}\left[\hat{\Delta}_{n, h}(\gamma)-\Delta(\gamma)\right]-\zeta_{n}(\gamma)\right|=o_{p}(1)
$$

Theorem 6 below shows that $\zeta_{n}(\cdot)$ converges to a zero mean Gaussian process and so does $\sqrt{n}\left[\hat{\Delta}_{n, h}(\cdot)-\Delta(\cdot)\right]$.

THEOREM 6. Let Assumptions 1-5 hold. Then

(a) $\zeta_{n}(\cdot) \stackrel{d}{\rightarrow} \mathcal{Z}(\cdot)$

(b) $\sqrt{n}\left[\hat{\Delta}_{n, h}(\cdot)-\Delta(\cdot)\right] \stackrel{d}{\rightarrow} \mathcal{Z}(\cdot)$, where $\mathcal{Z}$ is a zero mean Gaussian process on $\Gamma$ with covariance function

$\operatorname{cov}\left(\mathcal{Z}\left(\gamma_{1}\right), \mathcal{Z}\left(\gamma_{2}\right)\right)=4 \operatorname{cov}\left[\kappa_{1}\left(W ; \gamma_{1}\right), \kappa_{1}\left(W ; \gamma_{2}\right)\right] \equiv \sigma_{\Delta}\left(\gamma_{1}, \gamma_{2}\right)$

If $\mathrm{H}_{0}$ also holds, then

$$
T_{n}(\cdot) \equiv \sqrt{n} \hat{\Delta}_{n, h}(\cdot) \stackrel{d}{\rightarrow} \mathcal{Z}(\cdot)
$$

Let $M: \mathcal{C}(\Gamma) \rightarrow \mathbb{R}^{+}$be $\|\cdot\|_{\infty}$ continuous. Then the continuous mapping theorem (Billingsley, 1999, p. 20) implies that

$M\left[T_{n}(\cdot)\right] \stackrel{d}{\rightarrow} M[\mathcal{Z}(\cdot)]$

under the null hypothesis. For example, with $M\left[T_{n}(\cdot)\right]=\int_{\Gamma}\left[T_{n}(\gamma)\right]^{2} d \mu(\gamma)$, we have 


$$
\begin{aligned}
M_{n} & \equiv M\left[T_{n}(\cdot)\right]=\int_{\Gamma}\left[T_{n}(\gamma)\right]^{2} d \mu(\gamma) \\
& =n \int_{\Gamma}\left[\hat{\Delta}_{n, h}(\gamma)\right]^{2} d \mu(\gamma) \stackrel{d}{\rightarrow} \int_{\Gamma}[\mathcal{Z}(\gamma)]^{2} d \mu(\gamma)
\end{aligned}
$$

under $H_{0}$.

\subsection{Global and Local Alternatives}

The global alternatives for our conditional independence test can always be written as

$H_{a}: f_{Z}(z) f_{X Y Z}(x, y, z)-f_{Y Z}(y, z) f_{X Z}(x, z)=\alpha(x, y, z)$,

for some nontrivial and nonzero function $\alpha(x, y, z)$. Then under $H_{a}$, we have

$\Delta(\gamma)=\int \varphi\left(\tilde{w}^{\prime} \gamma\right) \alpha(x, y, z) d x d y d z$

This will be nonzero for essentially all $\gamma \in \Gamma$ provided that $\varphi$ is GCR. It follows from Theorem 6 that

$\lim _{n \rightarrow \infty} \operatorname{Pr}\left(M_{n}>c_{n}\right)=1$

for any critical value $c_{n}=o(n)$. That is, the test is consistent: as the sample size increases, the test will eventually detect the alternative $H_{a}$.

To construct a local alternative, we consider a mixture distribution of the form

$H_{a, n}: f_{X Y Z}(x, y, z)=\left[\left(1-\frac{c}{\sqrt{n}}\right) f_{Y \mid Z}(y \mid z)+\frac{c}{\sqrt{n}} \tilde{\alpha}(y \mid x, z)\right] f_{X Z}(x, z)$,

where $c$ is a constant and $\tilde{\alpha}(y \mid x, z)$ is a conditional density function of $\tilde{Y}$ given $(\tilde{X}, \tilde{Z})$ such that $\tilde{Y} \not \perp \tilde{X} \mid \tilde{Z}$. By construction, $\tilde{\alpha}(y \mid x, z)$ is a nontrivial function of $x$ and $z$. That is, the distribution of $W$ is a mixture of two distributions: one satisfies the null of conditional independence and the other does not. The mixing proportion is local to unity. Equivalently, we can rewrite the local alternative as

$H_{a, n}: f_{X Y Z}(x, y, z)=f_{Y \mid Z}(y, z) f_{X Z}(x, z)+\frac{\alpha(x, y, z)}{\sqrt{n}}$

for $\alpha(x, y, z)=c\left[\tilde{\alpha}(y \mid x, z)-f_{Y \mid Z}(y \mid z)\right] f_{X Z}(x, z)$. Since $\tilde{\alpha}(y \mid x, z)$ depends on $x, \tilde{\alpha}(y \mid x, z)-f_{Y \mid Z}(y \mid z)$ cannot be a zero function. Hence when $\varphi$ is GCR and $c>0$,

$\pi_{\varphi}(\gamma):=\int \varphi\left(\tilde{w}^{\prime} \gamma\right) \alpha(x, y, z) d x d y d z \neq 0$

for essentially all $\gamma \in \Gamma$. 
Under Assumptions 1-5 and the local alternative $H_{a, n}$, we can use the same arguments as in the proof of Theorem 6 to show that

$$
M_{n}=\int_{\Gamma}\left[T_{n}(\gamma)\right]^{2} d \mu(\gamma) \stackrel{d}{\rightarrow} \int_{\Gamma}\left[\mathcal{Z}(\gamma)+\pi_{\varphi}(\gamma)\right]^{2} d \mu(\gamma) .
$$

The essentially nonzero mean is the source of the power of the ICM test against the local alternative.

The above local alternative asymptotics can be used to guide the choice of the weighting function $\mu$. Using Mercer's theorem, we can represent the covariance kernel $\sigma_{\Delta}\left(\gamma_{1}, \gamma_{2}\right)$ as

$\sigma_{\Delta}\left(\gamma_{1}, \gamma_{2}\right)=\sum_{j=1}^{\infty} \lambda_{j} e_{j}\left(\gamma_{1}\right) e_{j}\left(\gamma_{2}\right)$

where $\lambda_{j}$ is the eigenvalue of the covariance kernel and $e_{j}(\cdot)$ is the corresponding eigen function such that $\int_{\Gamma} \sigma_{\Delta}\left(\gamma_{1}, \gamma_{2}\right) e_{j}\left(\gamma_{1}\right) d \gamma_{1}=\lambda_{j} e_{j}\left(\gamma_{2}\right) \cdot\left\{e_{j}(\cdot)\right\}$ also forms a set of complete orthonormal bases in $L^{2}(\Gamma)$. Boning and Sowell (1999) show that choosing $\mu$ to be the uniform density delivers a test with the greatest weighted average local power against the set of local alternatives whose limiting local mean functions $\left\{\pi_{\varphi}(\gamma)\right\}$ assign weights $\lambda_{j}^{2}$ to $\pm e_{j}(\gamma)$. This provides some theoretical justification of the uniform weighting, which is used in our simulation study.

\subsection{Calculating the Asymptotic Critical Values}

Under the null, $M_{n}$ has a limiting distribution given by a functional of a zero mean Gaussian process whose covariance function depends on the DGP. The asymptotic critical values thus depend on the DGP and cannot be tabulated. One could follow Bierens and Ploberger (1997) and obtain upper bounds for the asymptotic critical values. Here, we use the conditional Monte Carlo approach suggested by Hansen (1996) to simulate the asymptotic null distribution.

To apply this approach, we construct a process $T_{n}^{*}(\cdot)$, which follows the desired zero mean Gaussian process conditional on $\left\{W_{i}\right\}$. The desired conditional covariance function for $T_{n}^{*}$ is

$\operatorname{cov}\left[T_{n}^{*}\left(\gamma_{1}\right), T_{n}^{*}\left(\gamma_{2}\right) \mid\left\{W_{i}\right\}_{i=1}^{n}\right]=\frac{4}{n} \sum_{i=1}^{n} \hat{\kappa}_{h, 1}\left(W_{i} ; \gamma_{1}\right) \hat{\kappa}_{h, 1}\left(W_{i} ; \gamma_{2}\right) \equiv \hat{\sigma}_{\Delta}\left(\gamma_{1}, \gamma_{2}\right)$,

where

$\hat{\kappa}_{h, 1}\left(W_{i} ; \gamma\right)=(n-1)^{-1} \sum_{j=1, j \neq i}^{n} \kappa_{h, 2}\left(W_{i}, W_{j} ; \gamma\right)$.

It is straightforward to show that under Assumptions 1-5 and the null hypothesis, $\hat{\sigma}_{\Delta}\left(\gamma_{1}, \gamma_{2}\right) \stackrel{p}{\rightarrow} \sigma_{\Delta}\left(\gamma_{1}, \gamma_{2}\right)$. 
A typical $T_{n}^{*}(\cdot)$ is constructed by generating $\left\{\mathcal{V}_{i}\right\}_{i=1}^{n}$ as IID standard normal random variables independent of $\left\{W_{i}\right\}$ and setting

$T_{n}^{*}(\gamma)=\frac{2}{\sqrt{n}} \sum_{i=1}^{n} \hat{\kappa}_{h, 1}\left(W_{i} ; \gamma\right) \mathcal{V}_{i}$

Following the arguments similar to the proof of Theorem 2 in Hansen (1996), we can show that under the null hypothesis,

$M_{n}^{*}=\int_{\Gamma}\left[T_{n}^{*}(\gamma)\right]^{2} d \mu(\gamma) \stackrel{d}{\rightarrow} \int_{\Gamma}[\mathcal{Z}(\gamma)]^{2} d \mu(\gamma)$,

provided that Assumptions 1-5 hold. Simulation results show that the empirical pdf's of $M_{n}$ and $M_{n}^{*}$ are fairly close. To save space, we do not report the results here, but they are available in Huang (2009).

To approximate the distribution of $M_{n}$, we follow the steps below:

(i) generate $\left\{\mathcal{V}_{i b}\right\}_{i=1}^{n}$ IID $N(0,1)$ random variables;

(ii) set

$$
T_{n, b}^{*}(\gamma) \equiv \frac{2}{\sqrt{n}} \sum_{i=1}^{n} \hat{\kappa}_{h, 1}\left(W_{i} ; \gamma\right) \mathcal{V}_{i b}
$$

(iii) set $M_{n, b}^{*} \equiv M\left[T_{n, b}^{*}(\cdot)\right]=\int_{\Gamma}\left[T_{n, b}^{*}(\gamma)\right]^{2} d \mu(\gamma)$.

This gives a simulated sample $\left(M_{n, 1}^{*}, \ldots, M_{n, B}^{*}\right)$, whose empirical distribution should be close to the true distribution of the actual test statistic $M_{n}$ under the null. Then we can compute the proportion of simulated values that exceed $M_{n}$ to get the simulated asymptotic $p$ value. We reject the null hypothesis if the simulated $p$ value lies below the specified level for the test. As Hansen (1996) points out, $B$ is under the control of the econometrician and can be chosen sufficiently large to obtain a good approximation.

\section{MONTE CARLO EXPERIMENTS}

In this section, we perform some Monte Carlo simulation experiments to examine the finite sample performance of our conditional independence test.

For all simulations, we generate IID $\left\{\left(X_{i}, Y_{i}, Z_{i}\right)\right\}$. We choose $\varphi(\cdot)$ to be the standard normal PDF, and $k(u)$ be the sixth-order Gaussian kernel $(q=6)$. The number of replications for each experiment is 1,000 , and the number of replications for simulating $M_{n}^{*}$ is 999 .

\subsection{Level and Power Studies}

We consider three data generating processes. Under DGP 1, the sample $\left\{\left(X_{i}, Y_{i}, Z_{i}\right)\right\}$ is generated according to 
$Y=\theta X+Z+\varepsilon_{Y}$,

$X=Z+Z^{2}+\varepsilon_{X}$,

where

$\left(\begin{array}{l}\varepsilon_{X} \\ \varepsilon_{Y}\end{array}\right) \sim N\left(0,\left(\begin{array}{cc}\sigma_{X}^{2} & 0 \\ 0 & \sigma_{Y}^{2}\end{array}\right)\right)=N\left(0,\left(\begin{array}{ll}4 & 0 \\ 0 & 1\end{array}\right)\right)$

and

$Z \sim N\left(0, \sigma_{Z}^{2}\right)=N(0,3)$

When $\theta=0$, the null is true; otherwise the alternative holds.

DGP 2 is a modification of DGP 1 that focuses on the consequences of fattailed distributions. Under DGP 2, $\varepsilon_{X}$ and $\varepsilon_{Y}$ are proportional to the Student $t$ with 3 degrees of freedom:

$\varepsilon_{X} \sim 2 t_{3}, \varepsilon_{Y} \sim t_{3}, \varepsilon_{X} \perp \varepsilon_{Y}$

DGP 3 is another modification of DGP 1. Under this DGP, we allow skewness, choosing both $\varepsilon_{X}$ and $\varepsilon_{Y}$ to be centered chi-square distributions:

$\varepsilon_{X} \sim 2\left(\chi_{1}^{2}-1\right), \varepsilon_{Y} \sim\left(\chi_{1}^{2}-1\right), \varepsilon_{X} \perp \varepsilon_{Y}$

We transform each variable so that its range is comparable to the support of the GCR function $\varphi(\cdot)$. For the standard normal PDF, the support is the real line but the function is effectively zero out of the interval $[-4,4]$. We transform each variable to be supported on this interval. This can be achieved by using the transformation below:

$X_{i} \rightarrow \frac{8}{\pi} \arctan \left(\frac{X_{i}-\bar{X}}{\sqrt{\left(X_{i}-\bar{X}\right)^{2} /(n-1)}}\right)$.

We transform $Y_{i}$ and $Z_{i}$ analogously. The conditional independence test is then applied to the transformed data. We ignore the boundary bias here as our suggested bias-removing transformation does not give rise to qualitatively different results. Although any compact $\Gamma$ with a nonempty interior can be used, we take $\Gamma=[-1,1]^{4}$. This choice ensures that $\left\{\tilde{W}_{i}^{\prime} \gamma, \gamma \in \Gamma\right\}$ can take any value in the effective support of $\varphi(\cdot)$.

To compute the ICM statistic $M_{n}$, we need to compute the integral $\int_{\Gamma}\left[T_{n}(\gamma)\right]^{2} d \mu(\gamma)$ where $\mu$ is the uniform distribution. In the absence of a closedform expression, we recommend using the Monte Carlo integration method. For each simulation replication, we choose $100 \gamma_{s}$ 's randomly from the uniform distribution on $[-1,1]^{4}$ and approximate the integral by the average $\sum_{s=1}^{100} T_{n}^{2}\left(\gamma_{s}\right) / 100$. 
We have also tried using 50 random draws, but the results are effectively the same. Note that $T_{n}^{2}\left(\gamma_{s}\right)$ depends on the bandwidth parameter $h$. In our simulation experiments, we employ the data-driven bandwidth $\hat{h}\left(\gamma_{s}\right)$ in (27) with $h_{0}=$ $n^{-1 /\left[3\left(2 q+d_{z}\right)\right]}$ and $\tau=0.5$. We use different bandwidths for different $\gamma$ 's. Given the bandwidth $\hat{h}\left(\gamma_{s}\right)$, we compute the statistic $T_{n}^{2}\left(\gamma_{s}\right)$ as $T_{n}^{2}\left(\gamma_{s}\right)=n \hat{\Delta}_{n, \hat{h}\left(\gamma_{s}\right)}^{2}\left(\gamma_{s}\right)$. The average of $T_{n}^{2}\left(\gamma_{s}\right)$ gives us the ICM statistic $M_{n}$.

We study the finite sample size and power of the test against conditional mean dependence. We use

$\rho_{X, Y \mid Z}=\frac{\operatorname{cov}(X, Y \mid Z)}{\sigma_{X \mid Z} \sigma_{Y \mid Z}}=\frac{\theta \sigma_{X}^{2}}{\sigma_{X} \sqrt{\theta^{2} \sigma_{X}^{2}+\sigma_{Y}^{2}}}=\frac{4 \theta}{2 \sqrt{4 \theta^{2}+1}}$

to indicate the strength of the dependence between $X$ and $Y$, conditional on $Z$. Since both $X \mid Z$ and $Y \mid Z$ are normal under DGP 1, in this case $\rho_{X, Y \mid Z}$ fully captures the dependence between $X$ and $Y$, conditional on $Z$.

We plot the power of the tests for $\rho$ ranging from -0.9 to 0.9 . For this, we choose

$\theta=\frac{\rho_{X, Y \mid Z}}{2 \sqrt{\left(1-\rho_{X, Y \mid Z}^{2}\right)}}$ for $\rho_{X, Y \mid Z}=-0.9,-0.8, \ldots, 0.9$.

Figures 1-3 report the size and power properties of our GCR test. The size and power look fairly good for sample sizes as small as 100, and they look very good when the sample size reaches 200. When the sample size is small, the levels of

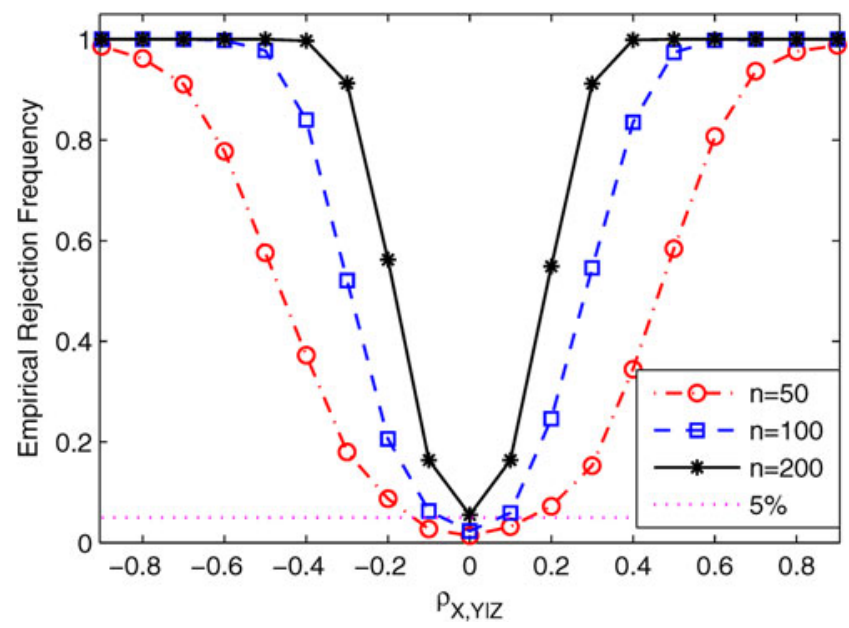

FIGURE 1. Power functions of the GCR test for DGP 1 with nominal size $5 \%$. 


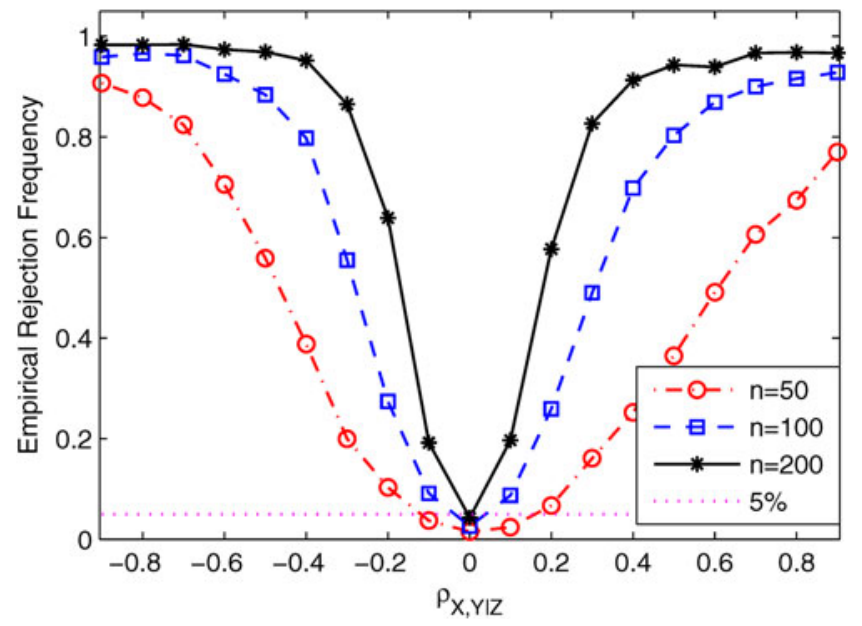

FIGURE 2. Power functions of the GCR test for DGP 2 with nominal size $5 \%$.

the tests approach their nominal value from below, delivering conservative tests. When the sample size increases to 200 , our tests become fairly accurate in size. The shape and location of the power curves are well expected. The power curves are also close to be symmetric, reflecting the equal capacity of our test to detect positive and negative dependence. The size and power of the test are close to each other across the three DGP's. This lends some support that the performance of our test is robust to the data distribution.

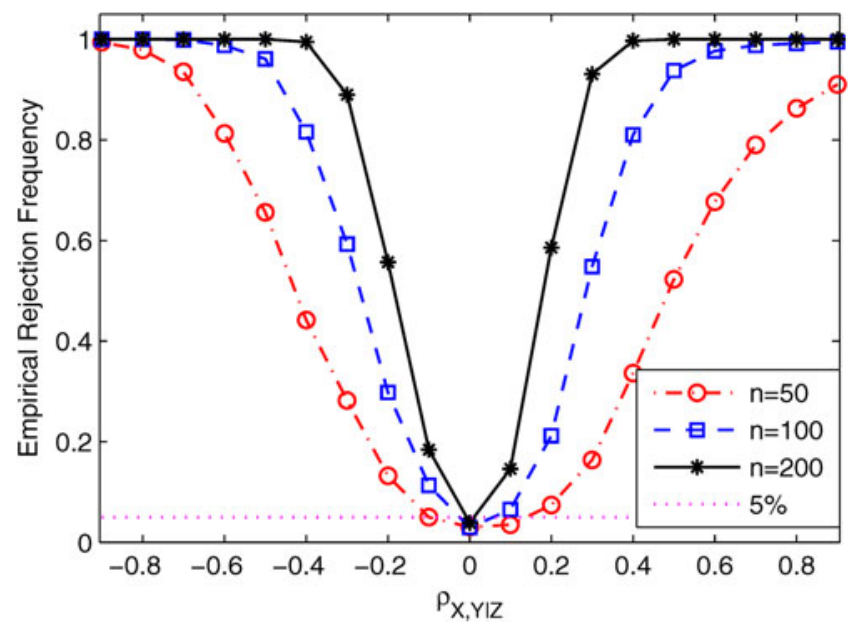

FIGURE 3. Power functions of the GCR test for DGP 3 with nominal size $5 \%$. 


\subsection{Comparison with Other Tests}

We now compare our GCR test with other conditional independence tests. Su and White's (2008) test essentially compares $f_{X Y Z} f_{Z}$ with $f_{X Z} f_{Y Z}$ and can detect local alternatives at the rate $n^{-1 / 2} h^{-d / 4}$. Su and White's (2007) test essentially compares $f_{Y \mid X, Z}$ with $f_{Y \mid Z}$ and can detect local alternatives at the rate $n^{-1 / 2} h^{-\left(d_{X}+d_{Z}\right) / 4}$. Our test compares integral transforms and can detect local alternatives at the rate $n^{-1 / 2}$. We first compare all three tests using DGP1. Figure 4 shows the power functions when the sample size is 100. It is clear that our GCR test outperforms the SW (2007) test, which in turn outperforms the SW (2008) test. More specifically, while our GCR test has almost the same empirical size as the SW (2007) test, it is more powerful than the SW (2007) test. The SW (2008) test is very conservative and has almost no power when $\rho$ is small in absolute value. That is, when the departure from the null is small, the SW (2008) test is less able to detect it, compared with our GCR test and the SW (2007) test.

Figure 5 shows the power functions when the sample size is increased to 200. We see that the power of our GCR test improves faster than the power of SW (2007), which again improves faster than the power of SW (2008). These results are consistent with the local alternative rate results.

Finally, we compare the power function of our GCR test with the tests proposed by LG (1997) and DG (2001, Sect. 5.2). Figure 6 reports the results for DGP 1 with $n=200$. We report only the results for the Cramer-von Mises type test for each method, as the results for the Kolmogorov-Smirnov type test are qualitatively similar. In the figure, "LG" and "DG" represent the Cramer-von Mises type tests of LG (1997) and DG (2001, Sect. 5.2), respectively. The figure

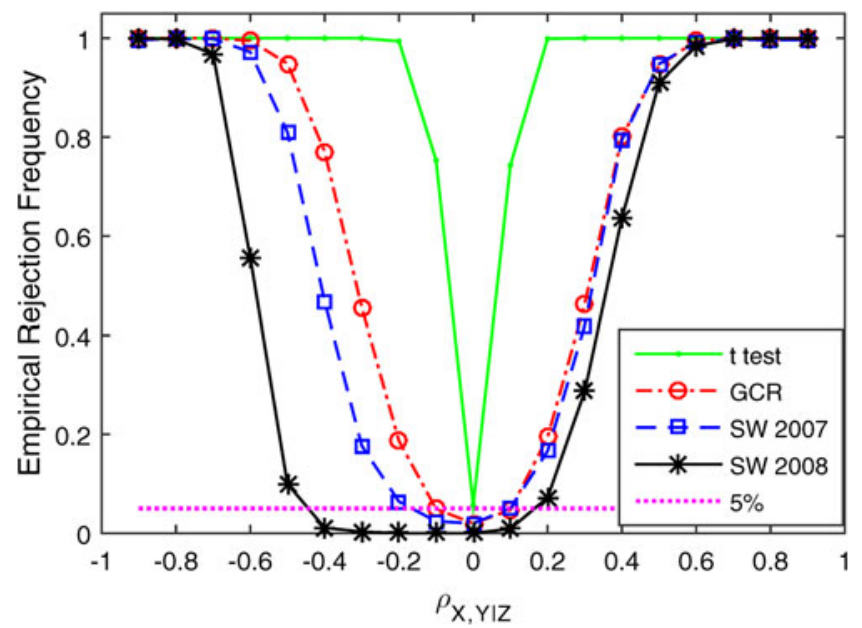

Figure 4. Power functions of the 5\% GCR test, SW (2007) test, and SW (2008) test under DGP1 with sample size 100. 


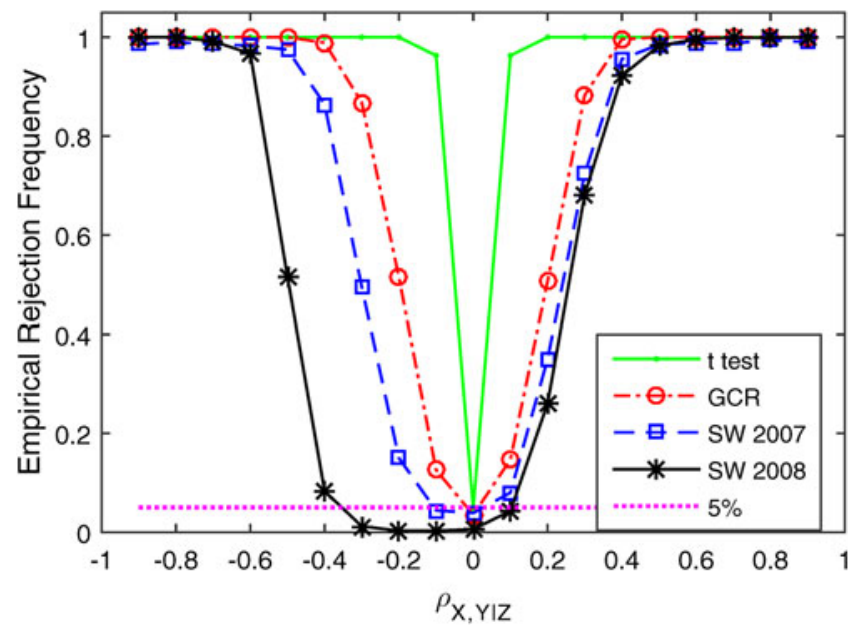

FIGURE 5. Power functions of the 5\% GCR test, SW (2007) test, and SW (2008) test under DGP1 with sample size 200.

demonstrates the clear advantage of our GCR test. It is as accurate in size as the LG test but more powerful than the latter test. The GCR test has better finite sample performances than the DG test in terms of both size accuracy and local power under the alternatives considered.

In all the figures, we also report the "gold standard" $t$-test. This is as good a test as one could want, in the sense that it is the parametric maximum likelihood test for $\theta=0$ in a correctly specified linear model. Although our test is not as powerful

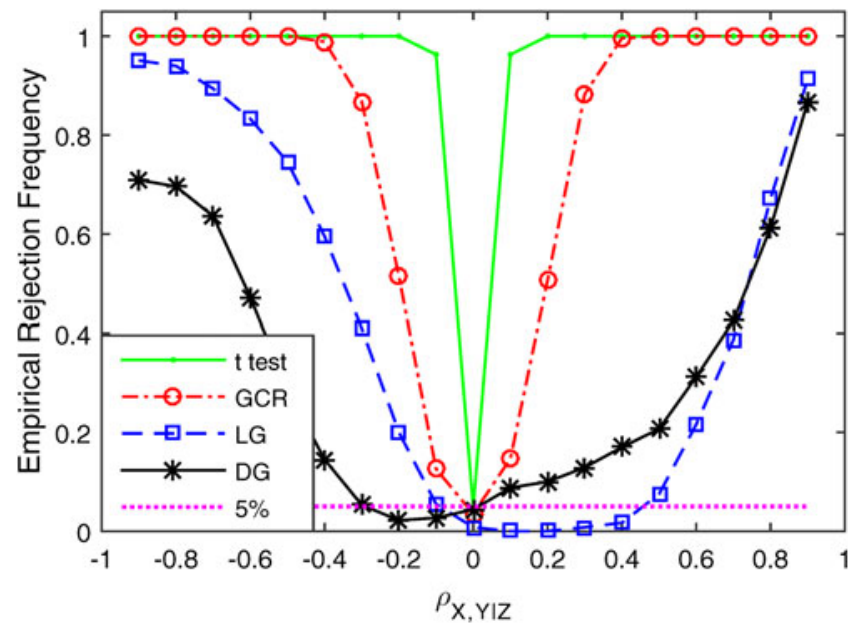

FIGURE 6. Power functions of the 5\% GCR test, LG (1997) test, and DG (2001) test under DGP1 with sample size 200. 
as the $t$-test, which is reasonable since our test is fully nonparametric, our GCR test does outperform all other nonparametric tests. On the other hand, the $t$-test measures only linear dependence. In the presence of nonlinear dependence, the $t$-test may be less powerful than the nonparametric tests. This is supported by simulation results not reported here.

\section{APPLICATION TO RETURNS TO SCHOOLING}

As stated in the introduction, one important application of tests for conditional independence is to test a key assumption identifying causal effects. In this section, we provide an example.

In the literature on returns to schooling, the most widely investigated structural equation is a Mincer (1974) type semilogarithmic human capital earnings function:

$\ln Y_{i}=\theta_{0}+\theta_{1} S_{i}+\theta_{2} E X P_{i}+\theta_{3} E X P_{i}^{2}+U_{i}$,

where the subscript $i$ indexes individuals, $\ln Y_{i}$ is log hourly wage, $S_{i}$ is years of completed schooling, $E X P_{i}$ is years of work experience, $E X P_{i}^{2}$ is work experience squared, and $U_{i}$ represents unobserved drivers of $\ln Y_{i}$, centered at zero. The effect of interest is $\theta_{1}$, the effect of an additional year of schooling on wage. In what follows, we drop the $i$ subscript.

Least squares estimates of the Mincer equation suffer from the well-known ability bias problem, which is caused by the dependence of schooling on unobserved ability. To make this explicit, let $U=A+\varepsilon$, where $A$ represents unobserved ability, and rewrite the Mincer equation as

$\ln Y=\theta_{0}+\theta_{1} S+\theta_{2} E X P+\theta_{3} E X P^{2}+A+\varepsilon$.

One method empirical researchers have adopted to address the ability bias issue is to find proxies $Z$ for ability, for example IQ or AFQT scores, and include these as regressors (e.g., Griliches and Mason, 1972; Griliches, 1977; Blackburn and Neumark, 1993). Now consider the regression of $\ln Y$ on $S, E X P$, and $Z$ :

$$
\begin{aligned}
\mu(S, E X P, Z) & =E(\ln Y \mid S, E X P, Z) \\
& =E\left(\theta_{0}+\theta_{1} S+\theta_{2} E X P+\theta_{3} E X P^{2}+A+\varepsilon \mid S, E X P, Z\right) \\
& =\theta_{0}+\theta_{1} S+\theta_{2} E X P+\theta_{3} E X P^{2}+E(A+\varepsilon \mid S, E X P, Z) \\
& =\theta_{0}+\theta_{1} S+\theta_{2} E X P+\theta_{3} E X P^{2}+E(A+\varepsilon \mid E X P, Z) .
\end{aligned}
$$

The last equality is justified by a conditional mean independence assumption,

$E(A+\varepsilon \mid S, E X P, Z)=E(A+\varepsilon \mid E X P, Z)$.

If this holds, then we have

$(\partial / \partial s) \mu(S, E X P, Z)=\theta_{1}$,

so that the effect of interest, $\theta_{1}$, is identified and can be consistently estimated. 
There is no reason a priori that the wage equation must have the specific Mincer form, however. More generally, one can consider a nonparametric specification $\ln Y=r(S, X, U)$,

where $r$ is an unknown function; $X$ contains observable factors determining wages, including $E X P$, as well as other factors like job tenure, region, sex, race, etc.; and $U=(A, \varepsilon)$.

An important effect of interest here is

$\phi_{1}(S, X, U)=(\partial / \partial s) r(S, X, U)$,

the marginal effect of schooling on wage. This effect depends on all drivers of wage, including unobservables, $U$, so $\phi_{1}(S, X, U)$ is not identifiable without further potentially strong restrictions. Nevertheless, just as in the linear case, it is possible to identify and estimate certain expectations of $\phi_{1}(S, X, U)$ given suitable ability proxies $Z$, as

$$
\begin{aligned}
(\partial / \partial s) \mu(s, x, z) & =(\partial / \partial s) E(\ln Y \mid S=s, X=x, Z=z) \\
& =E((\partial / \partial s) r(S, X, U) \mid S=s, X=x, Z=z) \\
& =E\left(\phi_{1}(s, X, U) \mid X=x, Z=z\right) \equiv \bar{\phi}_{1}(s, x, z) .
\end{aligned}
$$

The crucial condition justifying the third equality is conditional independence:

$$
(A, \varepsilon) \perp S \mid(X, Z)
$$

This is called a "conditional exogeneity" assumption by White and Chalak (2008). It implies the "ignorability" or "unconfoundedness" condition, also known as "selection on observables" in the literature, ensuring identification of causal effects.

Thus, if (36) holds, and even if the specific Mincer function (35) does not, we can still identify the average marginal effect of schooling $\bar{\phi}_{1}(s, x, z)$ and consistently estimate this by various methods. If (36) fails, then the marginal effect of interest is no longer identified (see, e.g., White and Chalak, 2008, Thm. 4.1).

We cannot test (36) directly, as $A$ and $\varepsilon$ are unobservable. However, following White and Chalak (2010), if we can observe $V$ such that

$$
\begin{aligned}
& V=f(A, \varepsilon, X, Z, \eta) \\
& \eta \perp S \mid(A, X, Z),
\end{aligned}
$$

where $f$ denotes some unknown function and $\eta$ is unobserved, then

$(A, \varepsilon) \perp S \mid(X, Z)$ implies $V \perp S \mid(X, Z)$.

Thus, we can test unconfoundedness by testing the implied condition

$H_{0}: V \perp S \mid(X, Z)$.

Equation (37) provides some guidance about how to choose $V$. The conditional independence requirement on $\eta$ is particularly plausible when $\eta$ is a measurement 
error, so that both $Z$ and $V$ could be error-laden proxies for ability. Here, we test (38) using data from the National Longitudinal Survey of Youth 1979 (NLSY 79). In particular, we use the data from survey year 2000 and restrict the sample to white males. ${ }^{1}$ We use the age-adjusted standardized AFQT in year 1980 as $Z$. $V$ includes math and verbal scores for preliminary scholastic aptitude tests from 1981 high school transcripts. To satisfy (37), we use years of schooling beyond high school as $S$, so that $V$ is not affected by $S$. $X$ includes actual work experience in survey year 2000 and total tenure with employer in survey year 2000.

To implement the test, we choose $\varphi(\cdot)$ to be the standard normal PDF, and let $k(\cdot)$ be the sixth-order Gaussian kernel. We choose $\gamma$ and other metaparameters as described in the Monte Carlo section. Applying our GCR test, we find that we do not reject the null hypothesis (38) at the 5\% level. Thus, we do not find evidence refuting the approach commonly used by empirical researchers.

\section{CONCLUDING REMARKS}

In this paper, we develop a flexible nonparametric test for conditional independence that is simple to implement, yet powerful. It is consistent against any deviation from the null and achieves local power at the parametric $n^{-1 / 2}$ rate, despite its nonparametric character. It is also very flexible as it allows for a rich class of GCR functions.

There are several useful directions for future research. First, we have assumed that the data are IID. But this is not essential for the results. We may straightforwardly extend the approach to a time-series framework, so that we could test, for example, nonlinear Granger causality. Another extension could be to modify the test so that it can be used when $Z$ contains both discrete and continuous variables. This is often relevant in applied microeconomics. This extension has been considered in Huang (2009, Chap. 3). A third direction is to further study the bandwidth selection problem. Here, we choose the bandwidth to minimize the MSE of $\hat{\Delta}_{n, h}(\gamma)$. Ideally, however, one should choose the bandwidth that optimizes the trade-off between size and power.

\section{NOTE}

1. To restrict the sample so that it is suitable for estimating a wage equation for survey year 2000 , we drop those who were enrolled in high school or college in survey year 2000, and we exclude those who were in active armed forces, self-employed, or working in a family business in survey year 2000. We also drop those whose hourly wage was not in the range $(\$ 1, \$ 1000]$.

\section{REFERENCES}

Andrews, D.W.K. (1994) Empirical process methods in econometrics. In R.F. Engle \& D.L. McFadden (eds.), Handbook of Econometrics, vol. IV, pp. 2248-2296. Elsevier.

Barnow, B.S., G.G. Cain, \& A.S. Goldberger (1981) Issues in the analysis of selectivity bias. In W.E. Stromsdorfer \& G. Farkas (eds.), Evaluation Studies Review Annual, vol. 5, pp. 43-59. Sage.

Bierens, H.J. (1982) Consistent model specification tests. Journal of Econometrics 20,105-134. 
Bierens, H.J. (1990) A consistent conditional moment test of functional form. Econometrica 58, 1443-1458.

Bierens, H.J. \& W. Ploberger (1997) Asymptotic theory of integrated conditional moment tests. Econometrica 65, 1129-1151.

Bierens, H.J. \& L. Wang (2012) Integrated conditional moment tests for parametric conditional distributions. Econometric Theory 28, 328-362.

Billingsley, P. (1999) Convergence of Probability Measures. Wiley.

Blackburn, M. \& D. Neumark (1993) Omitted-ability bias and the increase in the return to schooling. Journal of Labor Economics 11, 521-544.

Boning, W.B. \& F. Sowell (1999) Optimality for the integrated conditional moment test. Econometric Theory 15, 710-718.

Dawid, A.P. (1979) Conditional independence in statistical theory. Journal of the Royal Statistical Society, Series $B$ 41, 1-31.

Delgado, M. \& W. Gonzalez-Manteiga (2001) Significance testing in nonparametric regression based on the bootstrap. Annals of Statistics 29, 1469-1507.

Fernandes, M. \& R. Flores (1999) Tests for Conditional Independence, Markovian Dynamics and Noncausality. Discussion paper, European University Institute.

Geenens, G. (2014) Probit transformation for kernel density estimation on the unit interval. Journal of the American Statistical Association 109(505), 346-358.

Griliches, Z. (1977) Estimating the returns to schooling: Some econometric problems. Econometrica $45,1-22$.

Griliches, Z. \& W.M. Mason (1972) Education, income, and ability. The Journal of Political Economy 80(3), S74-S103.

Hansen, B.E. (1996) Inference when a nuisance parameter is not identified under the null hypothesis. Econometrica 64, 413-430.

Hoeffding, W. (1948) A class of statistics with asymptotically normal distribution. Annals of Mathematical Statistics 19, 293-325.

Huang, M. (2009) Essays on testing conditional independence. Ph.D. dissertation, University of California. Available at http://escholarship.org/uc/item/15t6n3h6.

Lee, A.J. (1990). U-statistics: Theory and Practice. CRC Press.

Li, Q. \& Y. Fan (2003) A kernel-based method for estimating additive partially linear models. Statistica Sinica 13, 739-762.

Li, Q. \& J.S. Racine (2007) Nonparametric Econometrics. Princeton University Press.

Linton, O. \& P. Gozalo (1997) Conditional Independence Restrictions: Testing and Estimation. Discussion Paper, Yale University Cowles Foundation for Research in Economics.

Mincer, J. (1974) Schooling, Experience, and Earnings. Columbia University Press.

Powell, J.L., J.H. Stock, \& T.M. Stoker (1989) Semiparametric estimation of index coefficients. Econometrica 57, 1403-1430.

Powell, J.L. \& T.M. Stoker (1996) Optimal bandwidth choice for density-weighted averages. Journal of Econometrics 75, 291-316.

Robinson, P.M. (1988) Root-N-consistent semiparametric regression. Econometrica 56, 931-954.

Song, K. (2009) Testing conditional independence via rosenblatt transforms. Annals of Statistics 37, 4011-4045.

Stinchcombe, M. \& H. White (1998) Consistent specification testing with nuisance parameters present only under the alternative. Econometric Theory 14, 295-324.

Su, L. \& H. White (2003) Testing Conditional Independence Via Empirical Likelihood. Discussion Paper, UCSD Department of Economics.

Su, L. \& H. White (2007) A consistent characteristic function-based test for conditional independence. Journal of Econometrics 141, 807-834.

Su, L. \& H. White (2008) A nonparametric hellinger metric test for conditional independence. Econometric Theory 24, 829-864.

Su, L. \& H. White (2010) Testing structural change in partially linear models. Econometric Theory 26, 1761-1806. 
White, H. \& K. Chalak (2008) Identifying Structural Effects in Nonseparable Systems Using Covariates. Discussion Paper, UCSD Department of Economics.

White, H. \& K. Chalak (2010) Testing a conditional form of exogeneity. Economics Letters 109, $88-90$.

\section{APPENDIX}

\section{A.1. Proofs of the Main Results}

Throughout the proofs, we use $C$ to denote a constant that may be different across different equations or lines.

Proof of Lemma 3. For the pointwise result, we use Assumption 1 and the theory of U-statistics to obtain

$$
\begin{aligned}
\operatorname{var}\left[\sqrt{n} R_{n, h}(\gamma)\right] & =\frac{2}{(n-1)} \operatorname{var}\left[\tilde{\kappa}_{h, 2}\left(W_{i}, W_{j}, \gamma\right)\right] \\
& \leq \frac{2}{(n-1)} \operatorname{var}\left[\kappa_{h, 2}\left(W_{i}, W_{j}, \gamma\right)\right] \leq \frac{2}{(n-1)} E\left[\kappa_{h, 2}^{2}\left(W_{i}, W_{j}, \gamma\right)\right] .
\end{aligned}
$$

So it suffices to show that $E\left[\kappa_{h, 2}^{2}\left(W_{i}, W_{j}, \gamma\right)\right]=o(n)$. But

$$
\begin{aligned}
\kappa_{h, 2}( & \left.W_{i}, W_{j}, \gamma\right) \\
= & \frac{1}{2}\left[\varphi\left(\gamma_{0}+X_{i}^{\prime} \gamma_{1}+Y_{i}^{\prime} \gamma_{2}+Z_{i}^{\prime} \gamma_{3}\right)-\varphi\left(\gamma_{0}+X_{i}^{\prime} \gamma_{1}+Y_{j}^{\prime} \gamma_{2}+Z_{i}^{\prime} \gamma_{3}\right)\right] K_{h}\left(Z_{i}-Z_{j}\right) \\
& +\frac{1}{2}\left[\varphi\left(\gamma_{0}+X_{j}^{\prime} \gamma_{1}+Y_{j}^{\prime} \gamma_{2}+Z_{j}^{\prime} \gamma_{3}\right)-\varphi\left(\gamma_{0}+X_{j}^{\prime} \gamma_{1}+Y_{i}^{\prime} \gamma_{2}+Z_{j}^{\prime} \gamma_{3}\right)\right] K_{h}\left(Z_{j}-Z_{i}\right),
\end{aligned}
$$

and so

$$
\begin{aligned}
E\left[\kappa_{h, 2}^{2}\left(W_{i}, W_{j}, \gamma\right)\right] & \\
\leq & E\left|\varphi\left(\gamma_{0}+X_{i}^{\prime} \gamma_{1}+Y_{i}^{\prime} \gamma_{2}+Z_{i}^{\prime} \gamma_{3}\right) K_{h}\left(Z_{i}-Z_{j}\right)\right|^{2} \\
& +E\left|\varphi\left(\gamma_{0}+X_{i}^{\prime} \gamma_{1}+Y_{j}^{\prime} \gamma_{2}+Z_{i}^{\prime} \gamma_{3}\right) K_{h}\left(Z_{i}-Z_{j}\right)\right|^{2} \\
& +E\left|\varphi\left(\gamma_{0}+X_{j}^{\prime} \gamma_{1}+Y_{j}^{\prime} \gamma_{2}+Z_{j}^{\prime} \gamma_{3}\right) K_{h}\left(Z_{j}-Z_{i}\right)\right|^{2} \\
& +E\left|\varphi\left(\gamma_{0}+X_{j}^{\prime} \gamma_{1}+Y_{i}^{\prime} \gamma_{2}+Z_{j}^{\prime} \gamma_{3}\right) K_{h}\left(Z_{j}-Z_{i}\right)\right|^{2} \\
\leq & 4 \varphi_{\max }^{2} E K_{h}^{2}\left(Z_{i}-Z_{j}\right),
\end{aligned}
$$

where $\varphi_{\max }=\sup _{\gamma \in \Gamma} \sup _{W \in[0,1]^{d}} \varphi\left(\tilde{W}^{\prime} \gamma\right)$, which is finite under Assumption 3. Using Assumption 2, we have

$$
\begin{aligned}
& E K_{h}^{2}\left(Z_{i}-Z_{j}\right) \\
& \quad=\int_{[0,1]^{d}} \frac{1}{h^{2 d_{Z}}}\left|K\left(\frac{z_{1}-z_{2}}{h}\right)\right|^{2} f_{Z}\left(z_{1}\right) f_{Z}\left(z_{2}\right) d z_{1} d z_{2} \\
& \quad \leq \int_{[0,1]^{d}} \frac{1}{h^{d_{Z}}}\left(\int K^{2}(u) f_{Z}\left(z_{2}+u h\right) d u\right) f_{Z}\left(z_{2}\right) d z_{2}
\end{aligned}
$$




$$
\begin{aligned}
\leq & \frac{1}{h^{d_{Z}}}\left(\int_{[0,1]^{d}} f_{Z}^{2}(z) d z\right)\left(\int K^{2}(u) d u\right) \\
& +\frac{\rho}{h^{d_{Z}-1}}\left(\int_{-\infty}^{\infty} f_{Z}(z) d z\right) \int K^{2}(u)\|u\| d u \\
= & \frac{1}{h^{d_{Z}}} E\left[f_{Z}(Z)\right]\left(\int K^{2}(u) d u\right)+\frac{1}{h^{d_{Z}-1}} \int K^{2}(u)\|u\| d u,
\end{aligned}
$$

where $\int$ is the integral over the support of $K(\cdot)$. It follows from Assumption 4 that $\int K^{2}(u) d u=\left(\int_{-\infty}^{\infty} k^{2}(v) d v\right)^{d_{Z}}<\infty$ and

$$
\begin{aligned}
& \int K^{2}(u)\|u\| d u \\
& \quad=\int_{-\infty}^{\infty} k^{2}\left(u_{1}\right) \cdots k^{2}\left(u_{d_{Z}}\right) \sqrt{u_{1}^{2}+\cdots+u_{d_{Z}}^{2}} d u_{1} \cdots d u_{d_{Z}} \\
& \quad \leq \sqrt{d_{Z}} \int k^{2}\left(u_{1}\right) \cdots k^{2}\left(u_{d_{Z}}\right) \max _{i=1, \ldots, d_{Z}}\left|u_{i}\right| d u_{1} \cdots d u_{d_{Z}} \\
& \quad=\sqrt{d_{Z}} \int k^{2}\left(u_{1}\right) \cdots k^{2}\left(u_{d_{Z}}\right)\left(\left|u_{1}\right|+\cdots+\left|u_{d_{Z}}\right|\right) d u_{1} \cdots d u_{d_{Z}} \\
& \quad=d_{Z} \sqrt{d_{Z}}\left(\int k^{2}(v)|v| d v\right)\left(\int k^{2}(v) d v\right)^{d_{Z}-1}<\infty .
\end{aligned}
$$

Therefore

$$
E K_{h}^{2}\left(Z_{i}-Z_{j}\right)=O\left(\frac{1}{h^{d}}\right) .
$$

Combining this with (A.1), we have, using Assumption 5(a):

$$
E\left|\kappa_{h, i, j}(\gamma)\right|^{2}=O\left(\frac{1}{h^{d_{Z}}}\right)=O\left(n \times \frac{1}{n h^{d_{Z}}}\right)=o(n) .
$$

This implies that $R_{n, h}(\gamma)=o_{p}(1 / \sqrt{n})$ pointwise for each $\gamma \in \Gamma$.

To show the uniformity result that $\sup _{\gamma \in \Gamma} R_{n, h}(\gamma)=o_{p}(1 / \sqrt{n})$, we employ the theory of U-processes. In particular, we apply Proposition 4 in DG (2001) with their $k=2$. The class of functions under consideration is $\mathcal{K}=$ $\left\{\kappa_{h, 2}\left(W_{i}, W_{j}, \gamma\right): \gamma \in \Gamma\right\}$. Since $\left|\kappa_{h, 2}\left(W_{i}, W_{j}, \gamma\right)\right| \leq 2 \varphi_{\max }\left|K_{h}\left(Z_{i}-Z_{j}\right)\right|$, we can use $\mathbb{K}\left(W_{i}, W_{j}\right)=2 \varphi_{\max }\left|K_{h}\left(Z_{i}-Z_{j}\right)\right|$ as the envelope function. As sets of linear functions whose subgraphs are half planes, both $\left\{\tilde{W}_{i} \gamma: \gamma \in \Gamma\right\}$ and $\left\{\tilde{W}_{i j} \gamma: \gamma \in \Gamma\right\}$ are VC-type. Under Assumption 3(b), it is clear that $\left\{\varphi\left(\tilde{W}_{i} \gamma\right): \gamma \in \Gamma\right\}$ and $\left\{\varphi\left(\tilde{W}_{i j} \gamma\right): \gamma \in \Gamma\right\}$ also are VC-type. Multiplying by a fixed function $K_{h}(\cdot)$ will not change their VC property and the associated VC characteristics. Therefore $\left\{\kappa_{h, 2}\left(W_{i}, W_{j}, \gamma\right): \gamma \in \Gamma\right\}$ is VC type with VC characteristics independent of $h$. Applying Proposition 4 in DG (2001), we have

$$
E \sup _{\gamma \in \Gamma}\left|\frac{n(n-1)}{n} R_{n, h}(\gamma)\right|^{2} \leq C E \mathbb{K}^{2}\left(W_{i}, W_{j}\right)
$$

for some constant $C$ that does not depend on $h$. But $E \mathbb{K}^{2}\left(W_{i}, W_{j}\right)=O\left(1 / h^{d_{Z}}\right)$, and so $E \sup _{\gamma \in \Gamma}\left|\sqrt{n} R_{n, h}(\gamma)\right|^{2}=O\left(1 /\left(n h^{d_{Z}}\right)\right)=o(1)$. As a result, $\sup _{\gamma \in \Gamma} \sqrt{n} R_{n, h}(\gamma)=$ $o_{p}(1)$. 
Proof of Lemma 4. Part (a). We first establish an expansion of $\int_{[0,1]}{ }^{d} K_{h}(u-$ z) $f_{Z}(u) d u$, starting with

$$
\begin{aligned}
\int_{[0,1]^{d} Z} & K_{h}(u-z) f_{Z}(u) d u \\
= & \int_{[0,1]^{d} Z} \frac{1}{h^{d_{Z}}} k\left(\frac{u_{1}-z_{1}}{h}\right) \cdots k\left(\frac{u_{d_{Z}}-z_{d_{Z}}}{h}\right) f_{Z}(u) d u_{1} \cdots d u_{d_{Z}} \\
= & \int_{-z_{1} / h}^{\left(1-z_{1}\right) / h} \cdots \int_{-z_{d_{Z}} / h}^{\left(1-z_{d_{Z}}\right) / h}\left[k\left(v_{1}\right) \cdots k\left(v_{d_{Z}}\right)\right. \\
& \prod_{\ell=1}^{d_{Z}}\left[\int_{-z_{\ell} / h}^{\left(1-z_{\ell}\right) / h} k\left(v_{\ell}\right) d v_{\ell}\left(z_{1}+v_{1} h, \ldots, z_{d_{Z}}+v_{d_{Z}} h\right)\right] d v_{1} \cdots d v_{d_{Z}}(z) \\
& +\sum_{0<|j| \leq q-1} h^{|j|} \frac{D^{j} f_{Z}(z)}{j !} \prod_{\ell=1}^{d_{Z}}\left[\int_{-z_{\ell} / h}^{\left(1-z_{\ell}\right) / h} k\left(v_{\ell}\right) v_{\ell}^{j_{\ell}} d v_{\ell}\right] \\
& +\sum_{|j|=q} h^{|j|} \frac{D^{j} f_{Z}(z)}{j !} \prod_{\ell=1}^{d_{Z}}\left[\int_{-z_{\ell} / h}^{\left(1-z_{\ell}\right) / h} k\left(v_{\ell}\right) v_{\ell}^{j_{\ell}} d v_{\ell}\right]+C_{K} h^{q+1},
\end{aligned}
$$

where

$$
\begin{aligned}
\left|C_{K}\right| & =\left|\sum_{|j|=q+1} \prod_{\ell=1}^{d_{Z}} \int_{-z_{\ell} / h}^{\left(1-z_{\ell}\right) / h} \frac{D^{j} f_{Z}(z+\tilde{v} h)}{j !} k\left(v_{\ell}\right) v_{\ell}^{j_{\ell}} d v_{\ell}\right| \\
& \leq\left[\max _{j:|j|=q+1} \max _{z \in[0,1]^{d}} D^{j} f_{Z}(z) \sum_{|j|=q+1} \frac{1}{j !} \prod_{\ell=1}^{d_{Z}} \int_{-\infty}^{\infty}\left|k\left(v_{\ell}\right) v_{\ell}^{j_{\ell}}\right| d v_{\ell} \leq C .\right.
\end{aligned}
$$

Here we have used Assumptions 2(a) and 4(b).

When $z_{\ell} \in\left[h^{\alpha}, 1-h^{\alpha}\right]$ for some $\alpha \in(0,1)$, we have

$$
\begin{aligned}
& \left(\int_{-z_{\ell} / h}^{\left(1-z_{\ell}\right) / h} k\left(v_{\ell}\right) d v_{\ell}\right) f_{Z}(z) \\
& \quad=f_{Z}(z)-\left(\int_{z_{\ell} / h}^{\infty} k\left(v_{\ell}\right) d v_{\ell}\right) f_{Z}(z)-\left(\int_{\left(1-z_{\ell}\right) / h}^{\infty} k\left(v_{\ell}\right) d v_{\ell}\right) f_{Z}(z) .
\end{aligned}
$$

But under Assumption 4(b), we have, for some constants $\tilde{C}$ and $C$,

$$
\left|\int_{z_{\ell} / h}^{\infty} k\left(v_{\ell}\right) d v_{\ell}\right| \leq \int_{h^{\alpha-1}}^{\infty}\left|k\left(v_{\ell}\right)\right| d v_{\ell} \leq \tilde{C} \int_{h^{\alpha-1}}^{\infty} \frac{1}{1+\left|v_{\ell}\right|^{\xi}} d v_{\ell} \leq C h^{(1-\alpha)(\xi-1)},
$$

and similarly $\left|\int_{\left(1-z_{\ell}\right) / h}^{\infty} k\left(v_{\ell}\right) d v_{\ell}\right| \leq C h^{(1-\alpha)(\xi-1)}$. Hence

$$
\left|\left(\int_{-z_{\ell} / h}^{\left(1-z_{\ell}\right) / h} k\left(v_{\ell}\right) d v_{\ell}\right) f_{Z}(z)-f_{Z}(z)\right| \leq C h^{(1-\alpha)(\xi-1)} .
$$


When $z_{\ell} \in\left[0, h^{\alpha}\right)$, we have, for some $z_{\ell}^{*} \in\left(0, z_{\ell}\right)$,

$$
\begin{aligned}
& \left|\left(\int_{-z_{\ell} / h}^{\left(1-z_{\ell}\right) / h} k\left(v_{\ell}\right) d v_{\ell}\right) f_{Z}(z)\right| \\
& =\left|\left(\int_{-z_{\ell} / h}^{\left(1-z_{\ell}\right) / h} k\left(v_{\ell}\right) d v_{\ell}\right) f_{Z}\left(z_{1}, \ldots, z_{\ell}, \ldots z_{d_{Z}}\right)\right| \\
& \leq \tilde{C}\left|\left(\int_{-z_{\ell} / h}^{\left(1-z_{\ell}\right) / h}\left|k\left(v_{\ell}\right)\right| d v_{\ell}\right) \frac{\left(z_{\ell}^{*}\right)^{q+1}}{(q+1) !}\right| \leq C h^{\alpha(q+1)},
\end{aligned}
$$

where we have used Assumption 2(b). Similarly when $z_{\ell} \in\left(1-h^{\alpha}, 1\right]$,

$$
\left(\int_{-z_{\ell} / h}^{\left(1-z_{\ell}\right) / h} k\left(v_{\ell}\right) d v_{\ell}\right) f_{Z}(z) \leq C h^{\alpha(q+1)} .
$$

If we choose $\alpha \in\left(\frac{q}{q+1}, 1-\frac{q}{q^{2}+q+1}\right)$, which is feasible, then

$$
\sup _{z \in[0,1]^{d} Z}\left|\left(\int_{-z_{\ell} / h}^{\left(1-z_{\ell}\right) / h} k\left(v_{\ell}\right) d v_{\ell}\right) f_{Z}(z)-f_{Z}(z)\right| \leq C h^{q+e}
$$

for some $e>0$. Repeating the above arguments for other elements of $z$, we obtain

$$
\sup _{z \in[0,1]^{d} Z}\left|\prod_{\ell=1}^{d_{Z}}\left[\int_{-z_{\ell} / h}^{\left(1-z_{\ell}\right) / h} k\left(v_{\ell}\right) d v_{\ell}\right] f_{Z}(z)-f_{Z}(z)\right| \leq C h^{q+e} .
$$

By the same argument, we can show that under Assumption 4 and 2(a)(b):

$$
\sup _{z \in[0,1]^{d} Z}\left|\sum_{j: 0<|j| \leq q-1} h^{|j|} \frac{D^{j} f_{Z}(z)}{j !} \prod_{\ell=1}^{d_{Z}} \int_{-z_{\ell} / h}^{\left(1-z_{\ell}\right) / h} k\left(v_{\ell}\right) v_{\ell}^{j_{\ell}} d v_{\ell}\right| \leq C h^{q+e}
$$

and

$$
\begin{aligned}
& \sup _{z \in[0,1]^{d} Z}\left|\sum_{|j|=q} h\right|^{j} \mid \frac{D^{j} f_{Z}(z)}{j !} \prod_{\ell=1}^{d_{Z}}\left[\int_{-z_{\ell} / h}^{\left(1-z_{\ell}\right) / h} k\left(v_{\ell}\right) v_{\ell}^{j_{\ell}} d v_{\ell}\right] \\
& -\frac{\mu_{q}}{q !}\left[\sum_{\ell=1}^{d_{Z}} \frac{\partial^{q} f_{Z}(z)}{\partial z_{\ell}^{q}}\right] h^{q} \mid \leq C h^{q+e},
\end{aligned}
$$

where

$\mu_{q}=\int v^{q} k(v) d v$.

We have therefore proved that

$$
\begin{aligned}
& \sup _{z \in[0,1]^{d} Z}\left|\int_{[0,1]^{d} Z} K_{h}(u-z) f_{Z}(u) d u-\left\{f_{Z}(z)+\frac{\mu_{q}}{q !}\left[\sum_{\ell=1}^{d_{Z}} \frac{\partial^{q} f_{Z}(z)}{\partial z_{\ell}^{q}}\right] h^{q}\right\}\right| \\
& \quad \leq C h^{q+e} .
\end{aligned}
$$


Using the above result, we have

$$
\begin{aligned}
E[\varphi & \left.\left(\gamma_{0}+X_{i}^{\prime} \gamma_{1}+Y_{i}^{\prime} \gamma_{2}+Z_{i}^{\prime} \gamma_{3}\right) K_{h}\left(Z_{i}-Z_{j}\right)\right] \\
& =E\left\{E\left[\varphi\left(X_{i}, Y_{i}, Z_{i} ; \gamma\right) K_{h}\left(Z_{j}-Z_{i}\right) \mid W_{i}\right]\right\} \\
& =E\left\{\varphi\left(X_{i}, Y_{i}, Z_{i} ; \gamma\right)\left[\int_{[0,1]^{d} Z} K_{h}\left(u-Z_{i}\right) f_{Z}(u) d u\right]\right\} \\
& =E \varphi\left(X_{i}, Y_{i}, Z_{i} ; \gamma\right)\left\{f_{Z}\left(Z_{i}\right)+\frac{\mu_{q}}{q !}\left[\sum_{\ell=1}^{d_{Z}} \frac{\partial^{q} f_{Z}\left(Z_{i}\right)}{\partial Z_{i \ell}^{q}}\right] h^{q}\right\}+o\left(h^{q}\right) \\
& =E\left[\varphi\left(X_{i}, Y_{i}, Z_{i} ; \gamma\right) f_{Z}\left(Z_{i}\right)\right]+h^{q} C_{1}(\gamma)+o\left(h^{q}\right),
\end{aligned}
$$

where

$$
C_{1}(\gamma) \equiv \frac{\mu_{q}}{q !} E\left\{\varphi\left(X_{i}, Y_{i}, Z_{i} ; \gamma\right)\left[\sum_{\ell=1}^{d_{Z}} \frac{\partial^{q} f_{Z}\left(Z_{i}\right)}{\partial Z_{i \ell}^{q}}\right]\right\}
$$

and the $o\left(h^{q}\right)$ term holds uniformly over $\gamma \in \Gamma$.

Next, let

$\psi(z ; \tilde{x}, \tilde{z}, \gamma)=\int_{[0,1]^{d_{Y}}} \varphi(\tilde{x}, y, \tilde{z} ; \gamma) f_{Y Z}(y, z) d y$

be a function of $z$ indexed by $(\tilde{x}, \tilde{z}, \gamma)$. Since $\varphi(\tilde{x}, y, \tilde{z} ; \gamma)$ and $f_{Y Z}(y, z)$ are bounded, we can exchange differentiation with integration to obtain

$D_{z}^{j}[\psi(z ; \tilde{x}, \tilde{z}, \gamma)]=\int_{[0,1]^{d_{Y}}} \varphi(\tilde{x}, y, \tilde{z} ; \gamma) D_{z}^{j}\left[f_{Y Z}(y, z)\right] d y$,

where $D_{z}^{j}[\cdot]$ is the partial differentiation operator with respect to $z$. So according to Assumption 2(a) and (d), $\psi(z ; \tilde{x}, \tilde{z}, \gamma)$ is $q+1$ times continuously differentiable with respect to $z$. Furthermore, under Assumption 3 and for $j$ with $|j|=q$, we have,

$$
\begin{aligned}
\sup _{x_{1}, z_{1}, \gamma} \sup _{\left\|z^{(1)}-z^{(2)}\right\| \leq \epsilon}\left|D_{z^{(1)}}^{j}\left[\psi\left(z^{(1)} ; \tilde{x}, \tilde{z}, \gamma\right)\right]-D_{z^{(2)}}^{j}\left[\psi\left(z^{(2)} ; \tilde{x}, \tilde{z}, \gamma\right)\right]\right| \\
=\sup _{x_{1}, z_{1}, \gamma} \sup _{\left\|z^{(1)}-z^{(2)}\right\| \leq \epsilon} \int_{[0,1]^{d Y_{Y}}} \varphi(\tilde{x}, y, \tilde{z} ; \gamma) \cdot \mid D_{z}^{j}\left[f_{Y Z}\left(y, z^{(1)}\right)\right] \\
\quad-D_{z}^{j}\left[f_{Y Z}\left(y, z^{(2)}\right)\right] \mid d y \\
\leq \varphi_{\max } \sup _{\left\|z^{(1)}-z^{(2)}\right\| \leq \epsilon} \int_{[0,1]^{d Y}}\left|D_{z}^{j}\left[f_{Y Z}\left(y, z^{(1)}\right)\right]-D_{z}^{j}\left[f_{Y Z}\left(y, z^{(2)}\right)\right]\right| d y \\
=\varphi_{\max } \int_{[0,1]^{d_{Y}}} \tilde{\rho}\left(\left\|z^{(1)}-z^{(2)}\right\|\right) d y \\
\leq \tilde{\rho} \varphi_{\max } \times\left\|z^{(1)}-z^{(2)}\right\|
\end{aligned}
$$

for some constant $\tilde{\rho}>0$. Therefore $\psi(z ; \tilde{x}, \tilde{z}, \gamma) \in \mathcal{G}_{q+1}\left([0,1]^{d_{X}+d_{Z}} \times \Gamma, \epsilon, \tilde{\rho} \varphi_{\max }\right)$. In addition, note that

$\psi(z ; \tilde{x}, \tilde{z}, \gamma)=\left[\int_{[0,1]^{d_{Y}}} \varphi(\tilde{x}, y, \tilde{z} ; \gamma) f_{Y \mid Z}(y \mid z) d y\right] f_{Z}(z)$ 
which, combined with Assumption 2(b), implies that $D_{z}^{j} \psi(\breve{z} ; \tilde{x}, \tilde{z}, \gamma)=0$ for all $\breve{z}$ on the boundary on $[0,1]^{d z}$. Given these two properties, we can follow the same steps in showing (A.3) to obtain

$$
\begin{aligned}
\int_{[0,1]^{d} Z} K_{h}(u-\tilde{z}) \psi(u ; \tilde{x}, \tilde{z}, \gamma) d u \\
=\psi(\tilde{z} ; \tilde{x}, \tilde{z}, \gamma)+\frac{\mu_{q}}{q !}\left[\left.\sum_{\ell=1}^{d_{Z}} \frac{\partial^{q} \psi(u ; \tilde{x}, \tilde{z}, \gamma)}{\partial u_{\ell}^{q}}\right|_{u=\tilde{z}}\right] h^{q}+o\left(h^{q}\right) \\
=\psi(\tilde{z} ; \tilde{x}, \tilde{z}, \gamma)+\frac{\mu_{q}}{q !}\left[\sum_{\ell=1}^{d_{Z}} \varphi(\tilde{x}, y, \tilde{z} ; \gamma) \frac{\partial^{q} f_{Y Z}(y, \tilde{z})}{\partial \tilde{z}_{\ell}^{q}} d y\right] h^{q}+o\left(h^{q}\right)
\end{aligned}
$$

uniformly over $\gamma \in \Gamma$ and $(\tilde{x}, \tilde{z}) \in[0,1]^{d_{X}+d_{Z}}$. Using this result, we have

$$
\begin{aligned}
E[\varphi & \left.\left(X_{i}, Y_{j}, Z_{i} ; \gamma\right) K_{h}\left(Z_{i}-Z_{j}\right)\right] \\
& =E\left\{\int K_{h}\left(u-Z_{i}\right)\left[\int \varphi\left(X_{i}, y, Z_{i} ; \gamma\right) f_{Y Z}(y, u) d y\right] d u\right\} \\
& =E\left\{\int K_{h}\left(u-Z_{i}\right) \psi\left(u ; X_{i}, Z_{i}, \gamma\right) d u\right\} \\
& =E \psi\left(Z_{i} ; X_{i}, Z_{i}, \gamma\right)+C_{2}(\gamma) h^{q}+o\left(h^{q}\right)
\end{aligned}
$$

uniformly over $\gamma \in \Gamma$ where

$$
C_{2}(\gamma)=\frac{\mu_{q}}{q !} E\left\{\sum_{\ell=1}^{d_{Z}} \int \varphi\left(X_{i}, y, Z_{i}\right) \frac{\partial^{q} f_{Y Z}\left(y, Z_{i}\right)}{\partial Z_{i \ell}^{q}} d y\right\} .
$$

By definition, $\psi\left(Z_{i} ; X_{i}, Z_{i}, \gamma\right)=g_{X Z}\left(X_{i}, Z_{i} ; \gamma\right)$. So

$$
E\left[\varphi\left(X_{i}, Y_{j}, Z_{i} ; \gamma\right) K_{h}\left(Z_{i}-Z_{j}\right)\right]=E g_{X Z}\left(X_{i}, Z_{i} ; \gamma\right)+C_{2}(\gamma) h^{q}+o\left(h^{q}\right)
$$

uniformly over $\gamma \in \Gamma$.

Let $C_{3}(\gamma) \equiv C_{1}(\gamma)-C_{2}(\gamma)$, then

$$
\begin{aligned}
\Delta_{h}(\gamma) \equiv & E\left[\hat{\Delta}_{n, h}(\gamma)\right] \\
= & E\left\{\left[\varphi\left(X_{i}, Y_{i}, Z_{i} ; \gamma\right) K_{h}\left(Z_{i}-Z_{j}\right)-\varphi\left(X_{i}, Y_{j}, Z_{i} ; \gamma\right) K_{h}\left(Z_{i}-Z_{j}\right)\right\}\right. \\
= & E\left[\varphi\left(X_{i}, Y_{i}, Z_{i}\right) f_{Z}\left(Z_{i}\right)\right]+C_{1}(\gamma) h^{q}+o\left(h^{q}\right) \\
& -\left\{E\left[g_{X Z}\left(X_{i}, Z_{i} ; \gamma\right)\right]+C_{2}(\gamma) h^{q}+o\left(h^{q}\right)\right\} \\
= & \Delta(\gamma)+C_{3}(\gamma) h^{q}+o\left(h^{q}\right)
\end{aligned}
$$

uniformly over $\gamma \in \Gamma$. It then follows that under Assumption 5(b)

$E\left[\hat{\Delta}_{n, h}(\gamma)\right]=\Delta(\gamma)+o\left(n^{-1 / 2}\right)$

uniformly over $\gamma \in \Gamma$. 
Part (b). By definition

$$
H_{n, h}(\gamma)=\frac{2}{n} \sum_{i=1}^{n} \tilde{\kappa}_{h, 1}\left(W_{i} ; \gamma\right)=\frac{2}{n} \sum_{i=1}^{n}\left\{\kappa_{h, 1}\left(W_{i} ; \gamma\right)-\Delta_{h}(\gamma)\right\},
$$

where $\kappa_{h, 1}\left(W_{i} ; \gamma\right)=E\left[\kappa_{h}\left(W_{i}, W_{j} ; \gamma\right) \mid W_{i}\right]$ for $j \neq i$. Using the same arguments in proving part (a), we have

$$
\sup _{\gamma \in \Gamma} \sup _{W_{i} \in[0,1]^{d}}\left|\kappa_{h, 1}\left(W_{i} ; \gamma\right)-\left[\kappa_{1}\left(W_{i} ; \gamma\right)+\frac{1}{2} B_{5}\left(X_{i}, Y_{i}, Z_{i} ; \gamma\right) h^{q}\right]\right| \leq C h^{q+e},
$$

where

$$
\begin{aligned}
\kappa_{1}\left(W_{i} ; \gamma\right) & \\
= & \frac{1}{2} \varphi\left(X_{i}, Y_{i}, Z_{i} ; \gamma\right) f_{Z}\left(Z_{i}\right)-\frac{1}{2} \int \varphi\left(X_{i}, y, Z_{i} ; \gamma\right) f_{Y Z}\left(y, Z_{i}\right) d y \\
& +\frac{1}{2} \int \varphi\left(x, y, Z_{i} ; \gamma\right) f_{X Y Z}\left(x, y, Z_{i}\right) d x d y \\
& -\frac{1}{2} \int \varphi\left(x, Y_{i}, Z_{i} ; \gamma\right) f_{X Z}\left(x, Z_{i}\right) d x,
\end{aligned}
$$

$B_{1}\left(X_{i}, Y_{i}, Z_{i} ; \gamma\right) \equiv \frac{\mu_{q}}{q !} \varphi\left(X_{i}, Y_{i}, Z_{i} ; \gamma\right) \sum_{\ell=1}^{d_{Z}} \frac{\partial^{q} f_{Z}\left(Z_{i}\right)}{\partial Z_{i \ell}^{q}}$,

$B_{2}\left(X_{i}, Z_{i} ; \gamma\right) \equiv \frac{\mu_{q}}{q !} \sum_{\ell=1}^{d_{Z}} \int \varphi\left(X_{i}, y, Z_{i} ; \gamma\right) \frac{\partial^{q} f_{Y Z}\left(y, Z_{i}\right)}{\partial Z_{i \ell}^{q}} d y$

$B_{3}\left(Z_{i} ; \gamma\right) \equiv \frac{\mu_{q}}{q !} \sum_{\ell=1}^{d_{Z}} \int \frac{\partial^{q}\left[\varphi\left(x, y, Z_{i} ; \gamma\right) f_{X Y Z}\left(x, y, Z_{i}\right)\right]}{\partial Z_{i \ell}^{q}} d x d y$,

$B_{4}\left(Y_{i}, Z_{i} ; \gamma\right) \equiv \frac{\mu_{q}}{q !} \sum_{\ell=1}^{d_{Z}} \int \frac{\partial^{q}\left[\varphi\left(x, Y_{i}, Z_{i} ; \gamma\right) f_{X Z}\left(x, Z_{i}\right)\right]}{\partial Z_{i \ell}^{q}} d x$

and

$$
\begin{aligned}
B_{5}\left(X_{i}, Y_{i}, Z_{i} ; \gamma\right)= & B_{1}\left(X_{i}, Y_{i}, Z_{i} ; \gamma\right)-B_{2}\left(X_{i}, Z_{i} ; \gamma\right) \\
& -B_{4}\left(Y_{i}, Z_{i} ; \gamma\right)+B_{3}\left(Z_{i} ; \gamma\right) .
\end{aligned}
$$

It is easy to see that $E \kappa_{1}\left(W_{i} ; \gamma\right)=\Delta(\gamma)$. So

$$
\begin{aligned}
H_{n, h}(\gamma)= & \frac{2}{n} \sum_{i=1}^{n}\left\{\kappa_{1}\left(W_{i} ; \gamma\right)+\frac{1}{2} B_{5}\left(X_{i}, Y_{i}, Z_{i} ; \gamma\right) h^{q}\right\}-\Delta_{h}(\gamma) \\
= & \frac{2}{n} \sum_{i=1}^{n}\left[\kappa_{1}\left(W_{i} ; \gamma\right)-E \kappa_{1}\left(W_{i} ; \gamma\right)\right]+\frac{1}{n} \sum_{i=1}^{n} B_{5}\left(X_{i}, Y_{i}, Z_{i} ; \gamma\right) h^{q} \\
& -\left(\Delta_{h}(\gamma)-\Delta(\gamma)\right)+o\left(h^{q}\right),
\end{aligned}
$$

where the $o\left(h^{q}\right)$ term holds uniformly over $\gamma \in \Gamma$. 
Since $B_{5}\left(X_{i}, Y_{i}, Z_{i} ; \gamma\right)$ is continuous in $\gamma, E \sup _{\gamma \in \Gamma}\left|B_{5}\left(X_{i}, Y_{i}, Z_{i} ; \gamma\right)\right|<\infty$, $\left(X_{i}, Y_{i}, Z_{i}\right)$ is IID, and $\Gamma$ is compact, we can use a standard textbook argument to show that a ULLN applies to $n^{-1} \sum_{i=1}^{n} B_{5}\left(X_{i}, Y_{i}, Z_{i} ; \gamma\right)$. That is, $\sup _{\gamma \in \Gamma}\left|n^{-1} \sum_{i=1}^{n} B_{5}\left(X_{i}, Y_{i}, Z_{i} ; \gamma\right)\right|=O(1)$. Combining this with part (a), we have

$$
\begin{aligned}
H_{n, h}(\gamma) & =\frac{2}{n} \sum_{i=1}^{n}\left\{\kappa_{1}\left(W_{i} ; \gamma\right)-E\left[\kappa_{1}\left(W_{i} ; \gamma\right)\right]\right\}+O_{p}\left(h^{q}\right) \\
& =\frac{2}{n} \sum_{i=1}^{n}\left\{\kappa_{1}\left(W_{i} ; \gamma\right)-E\left[\kappa_{1}\left(W_{i} ; \gamma\right)\right]\right\}+o_{p}\left(\frac{1}{\sqrt{n}}\right)
\end{aligned}
$$

uniformly over $\gamma \in \Gamma$.

Proof of Theorem 5. As a direct implication of Lemmas 3 and 4, we have

$$
\sqrt{n}\left[\hat{\Delta}_{n, h}\left(\Gamma_{s}\right)-\Delta\left(\Gamma_{s}\right)\right]=\frac{2}{n} \sum_{i=1}^{n}\left\{\kappa_{1}\left(W_{i} ; \Gamma_{s}\right)-E\left[\kappa_{1}\left(W_{i} ; \Gamma_{s}\right)\right]\right\}+o_{p}(1)
$$

uniformly over $\gamma \in \Gamma$. The asymptotic normality now follows by applying the LindebergLevy CLT.

If in addition $H_{0}$ holds, then $\Delta\left(\Gamma_{S}\right)=0$ and

$$
\begin{aligned}
\kappa_{1}\left(W_{i} ; \gamma\right)= & \frac{1}{2} \varphi\left(X_{i}, Y_{i}, Z_{i} ; \gamma\right) f_{Z}\left(Z_{i}\right)-\frac{1}{2} \int \varphi\left(X_{i}, y, Z_{i} ; \gamma\right) f_{Y Z}\left(y, Z_{i}\right) d y \\
& +\frac{1}{2} \int \varphi\left(x, y, Z_{i} ; \gamma\right) f_{X Y Z}\left(x, y, Z_{i}\right) d x d y \\
& -\frac{1}{2} \int \varphi\left(x, Y_{i}, Z_{i} ; \gamma\right) f_{X Z}\left(x, Z_{i}\right) d x
\end{aligned}
$$

(under $\left.H_{0}\right)=\frac{1}{2} E\left[\varphi\left(X_{i}, Y_{i}, Z_{i} ; \gamma\right) f_{Z}\left(Z_{i}\right) \mid X_{i}, Y_{i}, Z_{i}\right]$

$$
\begin{aligned}
& -\frac{1}{2} E\left[\varphi\left(X_{i}, Y_{i}, Z_{i} ; \gamma\right) f_{Z}\left(Z_{i}\right) \mid X_{i}, Z_{i}\right] \\
& +\frac{1}{2} E\left[\varphi\left(X_{i}, Y_{i}, Z_{i} ; \gamma\right) f_{Z}\left(Z_{i}\right) \mid Z_{i}\right] \\
& -\frac{1}{2} E\left[\varphi\left(X_{i}, Y_{i}, Z_{i} ; \gamma\right) f_{Z}\left(Z_{i}\right) \mid Y_{i}, Z_{i}\right] \\
= & \Lambda\left(W_{i} ; \gamma\right) .
\end{aligned}
$$

Thus, given $H_{0}$ we have

$\Omega(\ell, m)=4 E\left[\Lambda\left(W_{i} ; \gamma_{\ell}\right) \Lambda\left(W_{i} ; \gamma_{m}\right)\right]$.

Proof of Theorem 6. Given Lemmas 3 and 4, it suffices to prove part (a). Theorem 5 shows that for a finite number of $\gamma$ 's, $\left\{\zeta_{n}\left(\gamma_{1}\right), \zeta_{n}\left(\gamma_{2}\right), \ldots, \zeta_{n}\left(\gamma_{s}\right)\right\}$ is asymptotically normal. Also, $\gamma \in \Gamma \subset \mathbb{R}^{1+d}$ with $\Gamma$ a compact (hence totally bounded) set. To complete the proof, we need to show that $\zeta_{n}(\gamma)$ is stochastically equicontinuous (e.g., see Andrews, 1994). For this, we use Theorems 4-6 in Andrews (1994). In view of the definition of $\kappa_{1}\left(W_{i} ; \gamma\right)$ in (A.4) and Theorem 6 in Andrews (1994), we only need to verify that each of the four terms satisfies Ossiander's $L^{2}$ entropy condition. 
For the first term in (A.4), $\varphi\left(W_{i} ; \gamma\right) f_{Z}\left(Z_{i}\right)$ belongs to the type IV class if we can verify that

$$
E\left\{\left[f_{Z}\left(Z_{i}\right)\right]^{2} \sup _{\gamma_{1}:\left\|\gamma_{1}-\gamma\right\|<\nu}\left|\varphi\left(W_{i} ; \gamma_{1}\right)-\varphi\left(W_{i} ; \gamma\right)\right|^{2}\right\} \leq C v^{\psi}
$$

for any $\gamma \in \Gamma$, for any $v>0$ in a neighborhood of 0 , and for some finite constants $C>0$ and $\psi>0$. Under Assumption 3, $\varphi\left(W_{i} ; \gamma\right)$ is differentiable in $\gamma$. Given that

$E\left\|f_{Z}\left(Z_{i}\right) \sup _{\gamma \in \Gamma} \partial\left[\varphi\left(W_{i} ; \gamma\right) / \partial \gamma\right]\right\|^{2}<\infty$

and $\Gamma$ is bounded, we can show that (A.10) holds by the mean value theorem and CauchySchwarz inequality.

Similarly, we can show that the other three terms in $\kappa_{1}\left(W_{i} ; \gamma\right)$ also belong to the type IV class. Hence $\zeta_{n}(\cdot) \stackrel{d}{\rightarrow} \mathcal{Z}(\cdot)$.

\section{A.2. Standardization with Estimated Location and Scale Parameters}

In this subsection, we show that the estimation errors in the location and scale parameters do not affect the first order asymptotic properties of our GCR test.

Suppose that our raw data is $\left(\breve{X}_{i}, \breve{Y}_{i}, \breve{Z}_{i}\right)$. For notational simplicity, we assume that each of random variables $\breve{X}_{i}, \breve{Y}_{i}, \breve{Z}_{i}$ is a scalar. Let $\mu_{x}$ and $\sigma_{x}$ be the location and scale parameters of $\breve{X}_{i}$ and let $\hat{\mu}_{x}$ and $\hat{\sigma}_{x}$ be their sample or estimated versions. Define $\mu_{y}, \mu_{z}$, $\sigma_{y}$, and $\sigma_{z}$ and their sample versions similarly. Let

$$
\begin{gathered}
X_{i}=\Psi\left(\frac{\breve{X}_{i}-\mu_{x}}{\sigma_{x}}\right), \hat{X}_{i}=\Psi\left(\frac{\breve{X}_{i}-\hat{\mu}_{x}}{\hat{\sigma}_{x}}\right) \\
Y_{i}=\Psi\left(\frac{\breve{Y}_{i}-\mu_{y}}{\sigma_{y}}\right), \hat{Y}_{i}=\Psi\left(\frac{\breve{Y}_{i}-\hat{\mu}_{y}}{\hat{\sigma}_{y}}\right) \\
Z_{i}=\Psi\left(\frac{\breve{Z}_{i}-\mu_{z}}{\sigma_{z}}\right), \hat{Z}_{i}=\Psi\left(\frac{\breve{Z}_{i}-\hat{\mu}_{z}}{\hat{\sigma}_{z}}\right),
\end{gathered}
$$

where $\Psi$ is a function mapping $\mathbb{R}$ into a bounded set $[a, b]$. Then $\left(X_{i}, Y_{i}, Z_{i}\right) \in[a, b]^{3}$. Our asymptotic development so far has been based on $\left(X_{i}, Y_{i}, Z_{i}\right)$ which is not feasible in general, as we do not know the location and scale of each random variable. In practice we have to use the feasible version $\left(\hat{X}_{i}, \hat{Y}_{i}, \hat{Z}_{i}\right)$. Define $\breve{\Delta}_{n, h}(\gamma)$ in the same manner as $\hat{\Delta}_{n, h}(\gamma)$ but with $\left(X_{i}, Y_{i}, Z_{i}\right)$ replaced by $\left(\hat{X}_{i}, \hat{Y}_{i}, \hat{Z}_{i}\right)$, i.e.,

$$
\begin{aligned}
& \breve{\Delta}_{n, h}(\gamma) \\
& =\frac{1}{n(n-1)} \sum_{i, j}\left[\varphi\left(\gamma_{0}+\hat{X}_{i}^{\prime} \gamma_{1}+\hat{Y}_{j}^{\prime} \gamma_{2}+\hat{Z}_{i}^{\prime} \gamma_{3}\right)-\varphi\left(\gamma_{0}+\hat{X}_{i}^{\prime} \gamma_{1}+\hat{Y}_{j}^{\prime} \gamma_{2}+\hat{Z}_{i}^{\prime} \gamma_{3}\right)\right] \\
& \quad \times K_{h}\left(\hat{Z}_{j}-\hat{Z}_{i}\right) .
\end{aligned}
$$


The question is whether the estimation uncertainty in the location and scale parameters affect the asymptotic distribution of our GCR test. The answer is no, provided that the following additional assumptions hold.

\section{Assumption A.1.}

(a) $\Psi(\cdot)$ is three times differentiable with derivatives $\Psi^{(k)}(\cdot)$ satisfying $\sup _{u \in \mathbb{R}}\left|\Psi^{(k)}(u) u^{k}\right|<\infty$ for $k=1,2,3$.

(b) $\hat{\mu}_{v}-\mu_{v}=O_{p}(1 / \sqrt{n})$ and $\hat{\sigma}_{v}-\sigma_{v}=O_{p}(1 / \sqrt{n})$ for $v=x, y, z$.

(c) the kernel function $k(\cdot)$ is continuously differentiable with a bounded derivative $k^{(1)}(\cdot)$ and $\int_{-\infty}^{\infty}\left[k^{(1)}(u)\right]^{j} u^{k} d u<\infty$ for $j=1,2$ and $k=1,2,3$.

(d) $n h^{d_{Z}+1} \rightarrow \infty$.

All of the above assumptions are mild. Assumption A.1(a) is satisfied for $\Psi(x)=$ $\arctan (x)$ and the normal CDF. If $\mu_{v}$ and $\sigma_{v}$ are the mean and standard deviation respectively, then Assumption A.1(b) holds under some moment conditions. In the absence of enough moments, we can let $\mu_{v}$ and $\sigma_{v}$ be the median and interquartile range respectively, in which case Assumption A.1(b) can still hold under mild conditions. Assumption A.1(c) holds for the commonly used kernel functions. Assumption A.1(d) is given for the multivariate case. It holds for the bandwidth rule given in (27) when $q>d_{Z} / 2+1$. In particular, when $d_{Z}=1$, we only need $q \geq 2$, which holds for any symmetric kernel.

THEOREM A.1. Let Assumptions 1-A.1 hold. Then under the null and the local alternative given in (31), $\sqrt{n}\left[\breve{\Delta}_{n, h}(\gamma)-\hat{\Delta}_{n, h}(\gamma)\right]=o_{p}$ (1) uniformly over $\gamma \in \Gamma$.

Proof of Theorem A.1. We let

$$
\begin{aligned}
& G_{1 x}\left(\breve{X}_{i}\right) \equiv G_{1}\left(\breve{X}_{i} ; \mu_{x}, \sigma_{x}\right) \equiv \frac{\partial \Psi\left(\frac{\breve{X}_{i}-\mu_{x}}{\sigma_{x}}\right)}{\partial\left(\mu_{x}, \sigma_{x}\right)^{\prime}}=\Psi^{(1)}\left(\frac{\breve{X}_{i}-\mu_{x}}{\sigma_{x}}\right)\left(\begin{array}{c}
-\frac{1}{\sigma_{x}} \\
-\frac{\breve{X}_{i}-\mu_{x}}{\sigma_{x}^{2}}
\end{array}\right) \\
& G_{2 x}\left(\breve{X}_{i}\right) \equiv G_{2}\left(\breve{X}_{i} ; \mu_{x}, \sigma_{x}\right) \equiv \frac{\partial^{2} \Psi\left(\frac{\breve{X}_{i}-\mu_{x}}{\sigma_{x}}\right)}{\partial\left(\mu_{x}, \sigma_{x}\right) \partial\left(\mu_{x}, \sigma_{x}\right)^{\prime}} \\
& =\Psi^{(2)}\left(\frac{\breve{X}_{i}-\mu_{x}}{\sigma_{x}}\right)\left(\begin{array}{c}
-\frac{1}{\sigma_{x}} \\
-\frac{\breve{X}_{i}-\mu_{x}}{\sigma_{x}^{2}}
\end{array}\right)\left(-\frac{1}{\sigma_{x}},-\frac{\breve{X}_{i}-\mu_{x}}{\sigma_{x}^{2}}\right) \\
& +\Psi^{(1)}\left(\begin{array}{c}
\breve{X}_{i}-\mu_{x} \\
\sigma_{x}
\end{array}\right)\left(\begin{array}{cc}
0 & \frac{1}{\sigma_{x}^{2}} \\
\frac{1}{\sigma_{x}^{2}} & \frac{2\left(\breve{X}_{i}-\mu_{x}\right)}{\sigma_{x}^{3}}
\end{array}\right)
\end{aligned}
$$

and

$\xi_{n x}=\left(\begin{array}{c}\sqrt{n}\left(\hat{\mu}_{x}-\mu_{x}\right) \\ \sqrt{n}\left(\hat{\sigma}_{x}-\sigma_{x}\right)\end{array}\right)$.

Under Assumption A.1(a), the elements of $G_{1 v}(\cdot), G_{2 v}(\cdot)$ and the third derivatives of $\Psi\left(\left(x-\mu_{v}\right) / \sigma_{v}\right)$ with respect to $\mu_{v}$ and $\sigma_{v}$ for $v=x, y, z$ are bounded functions on $\mathbb{R}$. 
It follows from Assumption A.1(a) and (b) that

$\hat{X}_{i}-X_{i}=\frac{1}{\sqrt{n}} G_{1 x}\left(\breve{X}_{i}\right)^{\prime} \xi_{n x}+\frac{1}{n} \xi_{n x}^{\prime} G_{2 x}\left(\breve{X}_{i}\right) \xi_{n x}+O_{p}\left(\frac{1}{n \sqrt{n}}\right)$,

uniformly over $i$. Similarly,

$\hat{Y}_{i}-Y_{i}=\frac{1}{\sqrt{n}} G_{1 y}\left(\breve{Y}_{i}\right)^{\prime} \xi_{n y}+\frac{1}{n} \xi_{n y}^{\prime} G_{2 y}\left(\breve{Y}_{i}\right) \xi_{n y}+O_{p}\left(\frac{1}{n \sqrt{n}}\right)$

and

$\hat{Z}_{i}-Z_{i}=\frac{1}{\sqrt{n}} G_{1 z}\left(\breve{Z}_{i}\right)^{\prime} \xi_{n z}+\frac{1}{n} \xi_{n z}^{\prime} G_{2 z}\left(\breve{Z}_{i}\right) \xi_{n z}+O_{p}\left(\frac{1}{n \sqrt{n}}\right)$

uniformly over $i$.

Using Assumption A.1(d), we now have

$$
\begin{aligned}
& \sqrt{n} \breve{\Delta}_{n, h}(\gamma)-\sqrt{n} \hat{\Delta}_{n, h}(\gamma) \\
&=\frac{1}{n(n-1)} \sum_{i, j}\left[\varphi\left(\tilde{W}_{i}^{\prime} \gamma\right)-\varphi\left(\tilde{W}_{i j}^{\prime} \gamma\right)\right] \sqrt{n}\left[K_{h}\left(\hat{Z}_{j}-\hat{Z}_{i}\right)-K_{h}\left(Z_{j}-Z_{i}\right)\right] \\
& \quad+\frac{1}{n(n-1)} \sum_{i, j}\left\{\left[\varphi^{(1)}\left(\tilde{W}_{i}^{\prime} \gamma\right)\right]\right. \\
&\left.\quad \times \sqrt{n}\left[\left(\hat{X}_{i}-X_{i}\right)^{\prime} \gamma_{1}+\left(\hat{Y}_{i}-Y_{i}\right)^{\prime} \gamma_{2}+\left(\hat{Z}_{i}-Z_{i}\right)^{\prime} \gamma_{3}\right] K_{h}\left(\hat{Z}_{j}-\hat{Z}_{i}\right)\right\} \\
&-\frac{1}{n(n-1)} \sum_{i, j}\left\{\varphi(1)\left(\tilde{W}_{i j}^{\prime} \gamma\right)\right. \\
&\left.\times \sqrt{n}\left[\left(\hat{X}_{i}-X_{i}\right)^{\prime} \gamma_{1}+\left(\hat{Y}_{j}-Y_{j}\right)^{\prime} \gamma_{2}+\left(\hat{Z}_{i}-Z_{i}\right)^{\prime} \gamma_{3}\right] K_{h}\left(\hat{Z}_{j}-\hat{Z}_{i}\right)\right\}+o_{p}(1) \\
&= I_{1}(\gamma)+I_{2 x}(\gamma)+I_{2 y}(\gamma)+I_{2 z}(\gamma)+o_{p}(1)
\end{aligned}
$$

uniformly over $\gamma \in \Gamma$ where

$$
\begin{aligned}
& I_{1}(\gamma)=\frac{1}{n(n-1)} \sum_{i, j}\left[\varphi\left(\tilde{W}_{i}^{\prime} \gamma\right)-\varphi\left(\tilde{W}_{i j}^{\prime} \gamma\right)\right] \sqrt{n}\left[K_{h}\left(\hat{Z}_{j}-\hat{Z}_{i}\right)-K_{h}\left(Z_{j}-Z_{i}\right)\right], \\
& I_{2 x}(\gamma)=\frac{1}{n(n-1)} \sum_{i, j}\left[\varphi^{(1)}\left(\tilde{W}_{i}^{\prime} \gamma\right)-\varphi^{(1)}\left(\tilde{W}_{i j}^{\prime} \gamma\right)\right]\left[\sqrt{n}\left(\hat{X}_{i}-X_{i}\right)^{\prime} \gamma_{1}\right] K_{h}\left(\hat{Z}_{j}-\hat{Z}_{i}\right), \\
& I_{2 y}(\gamma)=\frac{1}{n(n-1)} \sum_{i, j}\left\{\left[\varphi^{(1)}\left(\tilde{W}_{i}^{\prime} \gamma\right) \sqrt{n}\left(\hat{Y}_{i}-Y_{i}\right)^{\prime} \gamma_{2}-\varphi^{(1)}\left(\tilde{W}_{i j} \gamma\right) \sqrt{n}\left(\hat{Y}_{j}-Y_{j}\right)^{\prime} \gamma_{2}\right]\right. \\
& \left.\quad \times K_{h}\left(\hat{Z}_{j}-\hat{Z}_{i}\right)\right\}, \\
& I_{2 z}(\gamma)=\frac{1}{n(n-1)} \sum_{i, j}\left[\varphi^{(1)}\left(\tilde{W}_{i}^{\prime} \gamma\right)-\varphi^{(1)}\left(\tilde{W}_{i j}^{\prime} \gamma\right)\right] \sqrt{n}\left(\hat{Z}_{i}-Z_{i}\right)^{\prime} \gamma_{3} K_{h}\left(\hat{Z}_{j}-\hat{Z}_{i}\right) .
\end{aligned}
$$


We first show that $\sup _{\gamma \in \Gamma}\left|I_{1}(\gamma)\right|=o_{p}(1)$. Noting that under Assumption A.1(c),

$$
\begin{aligned}
\sqrt{n} K_{h}\left(\hat{Z}_{j}-\hat{Z}_{i}\right) & \\
= & \frac{\sqrt{n}}{h} K\left(\frac{Z_{j}-Z_{i}}{h}+\frac{\left[G_{1 z}\left(\breve{Z}_{j}\right)-G_{1 z}\left(\breve{Z}_{i}\right)\right]^{\prime} \xi_{n z}}{\sqrt{n} h}\right. \\
& \left.\quad+\frac{\xi_{n z}^{\prime}\left[G_{2 z}\left(\breve{Z}_{j}\right)-G_{2 z}\left(\breve{Z}_{i}\right)\right] \xi_{n z}}{n h}+O_{p}\left(\frac{1}{n \sqrt{n} h}\right)\right) \\
= & \frac{\sqrt{n}}{h} K\left(\frac{Z_{j}-Z_{i}}{h}+\frac{\left[G_{1 z}\left(\breve{Z}_{j}\right)-G_{1 z}\left(\breve{Z}_{i}\right)\right]^{\prime} \xi_{n z}}{\sqrt{n} h}+\frac{\xi_{n z}^{\prime}\left[G_{2 z}\left(\breve{Z}_{j}\right)-G_{2 z}\left(\breve{Z}_{i}\right)\right] \xi_{n z}}{n h}\right) \\
& +O_{p}\left(\frac{1}{n h^{2}}\right) \\
= & \frac{\sqrt{n}}{h} K\left(\frac{Z_{j}-Z_{i}}{h}+\frac{\left[\tilde{G}_{1 z}\left(Z_{j}\right)-\tilde{G}_{1 z}\left(Z_{i}\right)\right]^{\prime} \xi_{n z}}{\sqrt{n} h}+\frac{\xi_{n z}^{\prime}\left[\tilde{G}_{2 z}\left(Z_{j}\right)-\tilde{G}_{2 z}\left(Z_{i}\right)\right] \xi_{n z}}{n h}\right) \\
& +o_{p}(1)
\end{aligned}
$$

uniformly over $\gamma \in \Gamma$ where

$\tilde{G}_{1 z}(Z):=G_{1 z}\left(\mu_{z}+\sigma_{z} \Psi^{-1}(Z)\right)$ and $\tilde{G}_{2 z}(Z):=G_{2 z}\left(\mu_{z}+\sigma_{z} \Psi^{-1}(Z)\right)$,

we have

$$
\begin{aligned}
\sqrt{n} K_{h}\left(\hat{Z}_{j}-\hat{Z}_{i}\right)-\sqrt{n} K_{h}\left(Z_{j}-Z_{i}\right) \\
=\frac{1}{h^{2}} K^{(1)}\left(\frac{Z_{j}-Z_{i}}{h}\right)\left[\tilde{G}_{1 z}\left(Z_{j}\right)-\tilde{G}_{1 z}\left(Z_{i}\right)\right]^{\prime} \xi_{n z} \\
\quad+\frac{1}{\sqrt{n} h^{2}} K^{(1)}\left(\frac{Z_{j}-Z_{i}}{h}\right) \xi_{n z}^{\prime}\left[\tilde{G}_{2 z}\left(Z_{j}\right)-\tilde{G}_{2 z}\left(Z_{i}\right)\right] \xi_{n z}+o_{p}(1)
\end{aligned}
$$

uniformly over $\gamma \in \Gamma$, where the $o_{p}(1)$ term follows from the Markov inequality. As a result

$$
I_{1}(\gamma)=I_{11, \xi}(\gamma)+I_{12, \xi}(\gamma)+I_{13, \xi}(\gamma)+o_{p}(1),
$$

uniformly over $\gamma \in \Gamma$ where $I_{11, \xi}(\gamma)=I_{11}^{\prime}(\gamma) \xi_{n z}$ and $I_{12, \xi}(\gamma)=\xi_{n z}^{\prime} I_{12}(\gamma) \xi_{n z}$ with

$$
\begin{aligned}
I_{11}(\gamma)=\frac{1}{n(n-1)} \sum_{i, j}\{ & {\left[\varphi\left(\tilde{W}_{i}^{\prime} \gamma\right)-\varphi\left(\tilde{W}_{i j}^{\prime} \gamma\right)\right] } \\
& \left.\times \frac{1}{h} K^{(1)}\left(\frac{Z_{j}-Z_{i}}{h}\right) \frac{\tilde{G}_{1 z}\left(Z_{j}\right)-\tilde{G}_{1 z}\left(Z_{i}\right)}{h}\right\}
\end{aligned}
$$


and

$$
\begin{aligned}
I_{12}(\gamma)=\frac{1}{n(n-1)} \sum_{i, j}\{ & {\left[\varphi\left(\tilde{W}_{i}^{\prime} \gamma\right)-\varphi\left(\tilde{W}_{i j}^{\prime} \gamma\right)\right] } \\
& \left.\times \frac{1}{\sqrt{n}} \frac{1}{h} K^{(1)}\left(\frac{Z_{j}-Z_{i}}{h}\right) \frac{\tilde{G}_{2 z}\left(Z_{j}\right)-G_{2 z}\left(\tilde{Z}_{i}\right)}{h}\right\} .
\end{aligned}
$$

Without loss of generality and for notational simplicity, we consider the case when only one of $\mu_{z}$ and $\sigma_{z}$ has to be estimated. In this case, all of $G_{1 z}(\cdot), G_{2}(\cdot)$, and $\xi_{n z}$ are scalars. Let

$\tilde{K}_{h}\left(Z_{j}, Z_{i}\right)=\frac{1}{h} K^{(1)}\left(\frac{Z_{j}-Z_{i}}{h}\right) \frac{\left[\tilde{G}_{1 z}\left(Z_{j}\right)-\tilde{G}_{1 z}\left(Z_{i}\right)\right]}{h}$,

and

$$
\begin{aligned}
\omega_{h}\left(W_{i}, W_{j}, \gamma\right)=\frac{1}{2} & {\left[\varphi\left(\tilde{W}_{i}^{\prime} \gamma\right)-\varphi\left(\tilde{W}_{i j}^{\prime} \gamma\right)\right] \tilde{K}_{h}\left(Z_{j}, Z_{i}\right) } \\
& +\frac{1}{2}\left[\varphi\left(\tilde{W}_{j}^{\prime} \gamma\right)-\varphi\left(\tilde{W}_{j i}^{\prime} \gamma\right)\right] \tilde{K}_{h}\left(Z_{i}, Z_{j}\right),
\end{aligned}
$$

we can rewrite $I_{11}(\gamma)$ as

$$
\begin{aligned}
I_{11}(\gamma) & =\frac{1}{n(n-1)} \sum_{i \neq j}\left[\varphi\left(\tilde{W}_{i}^{\prime} \gamma\right)-\varphi\left(\tilde{W}_{i j}^{\prime} \gamma\right)\right] \tilde{K}_{h}\left(Z_{j}, Z_{i}\right) \\
& =\frac{2}{n(n-1)} \sum_{i<j} \omega_{h}\left(W_{i}, W_{j}, \gamma\right)
\end{aligned}
$$

which is a $\mathrm{U}$ process. Letting

$\omega_{h, 1}(w ; \gamma)=E \omega_{h}\left(w, W_{j} ; \gamma\right)$

and using Hoeffding's H-decomposition, we have

$I_{11}(\gamma)=E I_{11}(\gamma)+\frac{2}{n} \sum_{i=1}^{n}\left[\omega_{h, 1}\left(W_{i} ; \gamma\right)-E \omega_{h, 1}\left(W_{i} ; \gamma\right)\right]+R_{n, h}^{\omega}(\gamma)$,

where

$$
\begin{aligned}
R_{n, h}^{\omega}(\gamma)= & \frac{2}{n(n-1)} \sum_{i<j}\left[\omega_{h}\left(W_{i}, W_{j}, \gamma\right)-\omega_{h, 1}\left(W_{i} ; \gamma\right)\right. \\
& \left.-\omega_{h, 1}\left(W_{j} ; \gamma\right)+E \omega_{h}\left(W_{i}, W_{j}, \gamma\right)\right] .
\end{aligned}
$$


We proceed to use the theory of $U$ processes to evaluate the order of $I_{11}(\gamma)$. To compute $E I_{11}(\gamma)$, we observe that for $\tilde{u}$ and $\tilde{u}^{*}$ between 0 and $u$,

$$
\begin{aligned}
& E \varphi\left(\tilde{W}_{i}^{\prime} \gamma\right) \tilde{K}_{h}\left(Z_{j}, Z_{i}\right) \\
& =E \int_{0}^{1} \varphi\left(X_{i}, Y_{i}, Z_{i}, \gamma\right) \frac{1}{h} K^{(1)}\left(\frac{z-Z_{i}}{h}\right) \frac{\left[\tilde{G}_{1 z}(z)-\tilde{G}_{1 z}\left(Z_{i}\right)\right]}{h} f_{Z}(z) d z \\
& =E \int_{-Z_{i} / h}^{\left(1-Z_{i}\right) / h} \varphi\left(X_{i}, Y_{i}, Z_{i}, \gamma\right) K^{(1)}(u) \frac{\left[\tilde{G}_{1 z}\left(Z_{i}+u h\right)-\tilde{G}_{1 z}\left(Z_{i}\right)\right]}{h} f_{Z}\left(Z_{i}+u h\right) d u \\
& =E \int_{-Z_{i} / h}^{\left(1-Z_{i}\right) / h}\left\{\varphi\left(X_{i}, Y_{i}, Z_{i}, \gamma\right) K^{(1)}(u) u\left[\tilde{G}_{1 z}^{(1)}\left(Z_{i}+\tilde{u}^{*} h\right)\right]\right. \\
& \left.\times\left[f_{Z}\left(Z_{i}\right)+f_{Z}^{\prime}\left(Z_{i}+\tilde{u} h\right) u h\right]\right\} d u \\
& =E \varphi\left(X_{i}, Y_{i}, Z_{i}, \gamma\right) \tilde{G}_{1 z}^{(1)}\left(Z_{i}\right) f_{Z}\left(Z_{i}\right)\left[\int_{-\infty}^{\infty} K^{(1)}(u) u d u\right]+O(h) \text {, }
\end{aligned}
$$

and

$$
\begin{aligned}
& E\left[\varphi\left(\tilde{W}_{i j}^{\prime} \gamma\right)\right] \tilde{K}_{h}\left(Z_{j}, Z_{i}\right) \\
& =E \int_{0}^{1} \int_{0}^{1} \varphi\left(X_{i, y}, Z_{i}, \gamma\right) \frac{1}{h} K^{(1)}\left(\frac{z-Z_{i}}{h}\right) \frac{\left[\tilde{G}_{1 z}(z)-\tilde{G}_{1 z}\left(Z_{i}\right)\right]}{h} f_{Y Z}(y, z) d z d y \\
& =E \int_{0}^{1} \int_{-Z_{i} / h}^{\left(1-Z_{i}\right) / h}\left\{\varphi\left(X_{i, y}, Z_{i}, \gamma\right)\right. \\
& \left.\times K^{(1)}(u) \frac{\left[\tilde{G}_{1 z}\left(Z_{i}+u h\right)-\tilde{G}_{1 z}\left(Z_{i}\right)\right]}{h} f_{Y Z}\left(y, Z_{i}+u h\right)\right\} d u d y \\
& =E \int_{0}^{1} \int_{-Z_{i} / h}^{\left(1-Z_{i}\right) / h}\left\{\varphi\left(X_{i, y}, Z_{i}, \gamma\right) K^{(1)}(u) u\left[\tilde{G}_{1 z}^{(1)}\left(Z_{i}+\tilde{u}^{*} h\right)\right]\right. \\
& \left.\times\left[f_{Y Z}\left(y, Z_{i}\right)+D_{z} f_{Y Z}\left(y, Z_{i}+\tilde{u} h\right) u h\right]\right\} d u d y \\
& =\left[E \int_{0}^{1} \varphi\left(X_{i}, y, Z_{i}, \gamma\right) \tilde{G}_{1 z}^{(1)}\left(Z_{i}\right) f_{Y Z}\left(y, Z_{i}\right) d y\right]\left[\int_{-\infty}^{\infty} K^{(1)}(u) u d u\right] \\
& +O(h) \text {, }
\end{aligned}
$$

where the $O(h)$ terms hold uniformly over $\gamma \in \Gamma$. Under the null hypothesis, the expectation in (A.11) becomes

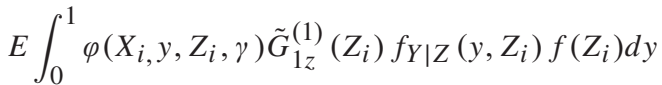

$$
\begin{aligned}
& =E \int \varphi\left(X_{i, y}, Z_{i}, \gamma\right) \tilde{G}_{1 z}^{(1)}\left(Z_{i}\right) f_{Y \mid Z, X}\left(y \mid Z_{i}, X_{i}\right) f\left(Z_{i}\right) d y \\
& =E \varphi\left(X_{i}, Y_{i}, Z_{i}, \gamma\right) \tilde{G}_{1 z}^{(1)}\left(Z_{i}\right) f_{Z}\left(Z_{i}\right) \text {. }
\end{aligned}
$$


Under the local alternative hypothesis, we have

$$
\frac{f_{X Y Z}(x, y, z)}{f_{X Z}(x, z)}=f_{Y \mid Z}(y, z)+\frac{\alpha(x, y, z)}{\sqrt{n} f_{X Z}(x, z)} \text {. }
$$

That is

$$
f_{Y \mid X, Z}(y \mid x, z)=f_{Y \mid Z}(y, z)+\frac{\alpha(x, y, z)}{\sqrt{n} f_{X Z}(x, z)} .
$$

So the expectation in (A.11) becomes

$$
\begin{aligned}
& E \int_{0}^{1} \varphi\left(X_{i,} y, Z_{i}, \gamma\right) \tilde{G}_{1 z}^{(1)}\left(Z_{i}\right) f_{Y \mid Z}\left(y, Z_{i}\right) f\left(Z_{i}\right) d y \\
& =E \int_{0}^{1} \varphi\left(X_{i, y}, Z_{i}, \gamma\right) \tilde{G}_{1 z}^{(1)}\left(Z_{i}\right)\left[f_{Y \mid X, Z}\left(y \mid X_{i}, Z_{i}\right)-\frac{\alpha\left(X_{i}, y, Z_{i}\right)}{\sqrt{n} f_{X Z}\left(X_{i}, Z_{i}\right)}\right] f\left(Z_{i}\right) d y \\
& =E \varphi\left(X_{i,} Y_{i}, Z_{i}, \gamma\right) \tilde{G}_{1 z}^{(1)}\left(Z_{i}\right) f_{Z}\left(Z_{i}\right) \\
& -E \int_{0}^{1}\left\{\varphi\left(X_{i}, y, Z_{i}, \gamma\right) \tilde{G}_{1 z}^{(1)}\left(Z_{i}\right)\left[\frac{\alpha\left(X_{i}, y, Z_{i}\right)}{\sqrt{n} f_{X Z}\left(X_{i}, Z_{i}\right) f_{Y \mid X, Z}\left(y \mid X_{i}, Z_{i}\right)}\right]\right. \\
& \left.\times f_{Y \mid X, Z}\left(y \mid X_{i}, Z_{i}\right)\right\} d y f\left(Z_{i}\right) \\
& =E \varphi\left(X_{i}, Y_{i}, Z_{i}, \gamma\right) \tilde{G}_{1 z}^{(1)}\left(Z_{i}\right) f_{Z}\left(Z_{i}\right) \\
& -\frac{1}{\sqrt{n}} E \varphi\left(X_{i, y}, Z_{i}, \gamma\right) \tilde{G}_{1 z}^{(1)}\left(Z_{i}\right) \frac{\alpha\left(X_{i}, Y_{i}, Z_{i}\right)}{f_{X Y Z}\left(X_{i}, Y, Z_{i}\right)} f\left(Z_{i}\right) .
\end{aligned}
$$

Therefore,

$\sup _{\gamma \in \Gamma}\left|E I_{11}(\gamma)\right|= \begin{cases}O(h)=o(1), & \text { under the null } \\ O(1 / \sqrt{n}+h)=o(1), & \text { under the local alternative }\end{cases}$

Following the same argument for proving Lemma 3, we can show that

$\sup _{\gamma \in \Gamma} R_{n, h}^{\omega}(\gamma)=O_{p}\left(\frac{1}{n}\left\{E\left[\tilde{K}_{h}\left(Z_{j}, Z_{i}\right)\right]^{2}\right\}^{1 / 2}\right)$.

But

$$
\begin{aligned}
& E \tilde{K}_{h}^{2}\left(Z_{j}, Z_{i}\right) \\
& =\frac{1}{h} \int_{0}^{1} \int_{0}^{1} \frac{1}{h}\left[K^{(1)}\left(\frac{z_{2}-z_{1}}{h}\right)\right]^{2} \frac{\left[\tilde{G}_{1 z}\left(z_{2}\right)-\tilde{G}_{1 z}\left(z_{1}\right)\right]^{2}}{h^{2}} f_{Z}\left(z_{1}\right) f_{Z}\left(z_{2}\right) d z_{1} d z_{2} \\
& =\frac{1}{h^{2}} \int_{0}^{1} \int_{z_{1} / h}^{1-z_{1} / h}\left\{\left[K^{(1)}(u)\right]^{2}\right. \\
& \left.\times \frac{\left[\tilde{G}_{1 z}\left(z_{1}+u h\right)-\tilde{G}_{1 z}\left(z_{1}\right)\right]^{2}}{h} f_{Z}\left(z_{1}\right) f_{Z}\left(z_{1}+u h\right)\right\} d u d z_{1}
\end{aligned}
$$


and hence $\sup _{\gamma \in \Gamma}\left|R_{n, h}^{\omega}(\gamma)\right|=O_{p}(1 /(n h))=o_{p}(1)$.

By definition,

$$
\begin{aligned}
\omega_{h, 1}\left(W_{i} ; \gamma\right)= & \frac{1}{2} E\left\{\left[\varphi\left(X_{i}, Y_{i}, Z_{i} ; \gamma\right)-\varphi\left(X_{i}, Y_{j}, Z_{i} ; \gamma\right)\right] \tilde{K}_{h}\left(Z_{j}, Z_{i}\right) \mid W_{i}\right\} \\
& +\frac{1}{2}\left\{E\left[\varphi\left(X_{j}, Y_{j}, Z_{j} ; \gamma\right)-\varphi\left(X_{j}, Y_{i}, Z_{j} ; \gamma\right)\right] \tilde{K}_{h}\left(Z_{j}, Z_{i}\right) \mid W_{i}\right\} .
\end{aligned}
$$

Note that

$$
\begin{aligned}
E\{[\varphi & \left.\left.\left(X_{i}, Y_{i}, Z_{i} ; \gamma\right)\right] \tilde{K}_{h}\left(Z_{j}, Z_{i}\right) \mid W_{i}\right\} \\
& =\int_{0}^{1}\left[\varphi\left(X_{i}, Y_{i}, Z_{i} ; \gamma\right)\right] \frac{1}{h} K^{(1)}\left(\frac{z-Z_{i}}{h}\right) \frac{\left[\tilde{G}_{1 z}(z)-\tilde{G}_{1 z}\left(Z_{i}\right)\right]}{h} f_{Z}(z) d z \\
& =\int_{-Z_{i} / h}^{1-Z_{i} / h}\left[\varphi\left(X_{i}, Y_{i}, Z_{i} ; \gamma\right)\right] K^{(1)}(u) \frac{\left[\tilde{G}_{1 z}\left(Z_{i}+u h\right)-\tilde{G}_{1 z}\left(Z_{i}\right)\right]}{h} f_{Z}\left(Z_{i}+u h\right) d u \\
& =\left[\varphi\left(X_{i}, Y_{i}, Z_{i} ; \gamma\right)\right] f_{z}\left(Z_{i}\right) \tilde{G}_{1 z}^{(1)}\left(Z_{i}\right)\left[\int_{-\infty}^{\infty} K^{(1)}(u) u d u\right]+O_{p}(h)
\end{aligned}
$$

uniformly over $i$ and $\gamma \in \Gamma$. Following the same steps, we can approximate other conditional expectations in $\omega_{h, 1}\left(W_{i} ; \gamma\right)$ and obtain

$\sup _{\gamma \in \Gamma W_{i} \in[0,1]^{d}}\left|\omega_{h, 1}\left(W_{i} ; \gamma\right)-\omega_{1}\left(W_{i} ; \gamma\right)\right|=O_{p}(h)$,

where

$\omega_{1}\left(W_{i} ; \gamma\right)=\kappa_{1}\left(W_{i} ; \gamma\right) \tilde{G}_{1 z}^{(1)}\left(Z_{i}\right)\left[\int_{-\infty}^{\infty} K^{(1)}(u) u d u\right]$

and $\kappa_{1}\left(W_{i} ; \gamma\right)$ is defined in (A.4). Using this result and a uniform law of large numbers, we have

$$
\begin{aligned}
& \frac{2}{n} \sum_{i=1}^{n} {\left[\omega_{h, 1}\left(W_{i} ; \gamma\right)-E \omega_{h, 1}\left(W_{i} ; \gamma\right)\right] } \\
& \quad=\frac{2}{n} \sum_{i=1}^{n}\left[\omega_{1}\left(W_{i} ; \gamma\right)-E \omega_{1}\left(W_{i} ; \gamma\right)\right]+O_{p}(h)=o_{p}(1) .
\end{aligned}
$$

This, combined with $\sup _{\gamma \in \Gamma}\left|E I_{11}\right|=o(1)$ and $\sup _{\gamma \in \Gamma}\left|R_{n, h}^{\omega}(\gamma)\right|=o_{p}(1)$, yields $\sup _{\gamma \in \Gamma}\left|I_{11}(\gamma)\right|=o_{p}(1)$. Similarly, we can show that $\sup _{\gamma \in \Gamma}\left|I_{12}(\gamma)\right|=o_{p}(1)$. So $\sup _{\gamma \in \Gamma}\left|I_{1}(\gamma)\right|=o_{p}(1)$.

Following the same arguments, we can show that $\sup _{\gamma \in \Gamma}\left|I_{2 v}(\gamma)\right|=o_{p}(1)$ for $v=x, y, z$. We have therefore proved that

$$
\sup _{\gamma \in \Gamma}\left|\sqrt{n} \breve{\Delta}_{n, h}(\gamma)-\sqrt{n} \hat{\Delta}_{n, h}(\gamma)\right|=o_{p}(1)
$$

as desired. 